CUBO A Mathematical Journal

Vol.12, No 02, (97-121). June 2010

\title{
The tree of primes in a field
}

\author{
WOLFGANG RUMP \\ Institute for Algebra and Number Theory, \\ University of Stuttgart, \\ Pfaffenwaldring 57, D-70550 Stuttgart, Germany \\ email: rump@mathematik. uni-stuttgart. de
}

Dedicated to B. V. M.

\begin{abstract}
The product formula of algebraic number theory connects finite and infinite primes in a stringent way, a fact, while not hard to be checked, that has never ceased to be tantalizing. We propose a new concept of prime for any field and investigate some of its properties. There are algebraic primes, corresponding to valuations, such that every prime contains a largest algebraic one. For a number field, this algebraic part is zero just for the infinite primes. It is shown that the primes of any field form a tree with a kind of self-similar structure, and there is a binary operation on the primes, unexplored even for the rationals. Every prime defines a topology on the field, and each compact prime gives rise to a unique Haar measure, playing an essential part in the product formula.
\end{abstract}

\section{RESUMEN}

La fórmula producto de la Teoría de Números Algebraicos conecta primos finitos e infinitos de una formula estricta, un hecho no difícil de ser verificado, es que nunca cesa de ser estudiado. Nosotros proponemos un nuevo concepto de primos para cualquier cuerpo e investigamos algunas de sus propiedades. Hay primos algebraicos, correspondientes a valuaciones, talque todo primo contiene un primo algebraico mayor. Para un número de cuerpos, esta parte algebraica es cero solamente para primos infinitos. Es demostrado que los primos de cualquier cuerpo forman un árbol con una clase de estructura auto-similar, y 
hay una operación binaria sobre los primos inexplorada incluso para los racionales. Todo primo define una topología sobre el cuerpo, y todo primo compacto da origen a una única medida de Haar, jugando rol esencial en la fórmula producto.

Key words and phrases: prime, valuation, product formula.

AMS (MOS) Subj. Class.: Primary: 11S15, 11N80, 12J20, 13A18. Secondary: 28C10

Let $K$ be a field, and let $p \neq 0$ be a polynomial in $K[x]$. Assume first that $p$ is normed and decomposes into powers of distinct linear factors:

$$
p=\left(x-b_{1}\right)^{e_{1}} \cdots\left(x-b_{r}\right)^{e_{r}} .
$$

Then $p$ is completely determined by the function $e: K \rightarrow \mathbb{Z}$ with $e\left(b_{i}\right):=e_{i}$ and $e(b):=0$ for all $b \notin\left\{b_{1}, \ldots, b_{r}\right\}$. Furthermore, $e(b) \geqslant 0$ for all $b \in K$. The same holds for rational functions $p=\frac{g}{h} \in K(x)$ if $g, h \in K[x]$ are normed and decompose into linear factors. Here $e$ may also attain negative values in $\mathbb{Z}$.

To complete the picture, replace $K$ by the projective line $\mathbb{P}^{1}(K):=K \cup\{\infty\}$. If $K=\mathbb{R}$, we can calculate the limit of $p$ for $x \rightarrow \infty$ : In case that $\operatorname{deg} p:=\operatorname{deg} g-\operatorname{deg} h<0$, we have $\lim _{x \rightarrow \infty} p(x)=0$, and $-\operatorname{deg} p$ can be regarded as the multiplicity of $\infty \in \mathbb{P}^{1}(K)$ as a zero of $p$. If $\operatorname{deg} p>0$, then $p$ has a pole in $\infty$, i. e. a zero with negative multiplicity $-\operatorname{deg} p$. Therefore, we set

$$
e(\infty):=-\operatorname{deg} p,
$$

and this definition makes sense for an arbitrary field $K$. The function $e$ vanishes almost everywhere, and

$$
\sum_{b \in \mathbb{P}^{1}(K)} e(b)=0
$$

In general, instead of (1), a polynomial $p$ is of the form

$$
p=a \cdot p_{1}^{e_{1}} \cdots p_{r}^{e_{r}}
$$

with $a \in K^{\times}$and different irreducible polynomials $p_{1}, \ldots, p_{r}$. Then the $p_{i}$ take the rôle of the points $b_{i} \in \mathbb{P}^{1}(K)$. However, $K$ can be extended such that every $p_{i}$ splits into $\operatorname{deg} p_{i}$ linear factors. To take this into account, a weight $f\left(p_{i}\right):=\operatorname{deg} p_{i}$ is attached to the point $p_{i}$, i. e. $p_{i}$ counts $f\left(p_{i}\right)$-fold. At infinity $\infty$ we still have $e(\infty)=-\operatorname{deg} p$, and $f(\infty):=1$. So Eq. (3) turns into

$$
\sum_{i=1}^{r} e\left(p_{i}\right) f\left(p_{i}\right)+e(\infty) f(\infty)=0
$$

In other words, every point $p_{i}$ has two invariants: the multiplicity $e\left(p_{i}\right)$, that is, the ramification order, and the weight $f\left(p_{i}\right)$, called the inertial degree.

Historically, the analogy between function fields and the field $\mathbb{Q}$ of rationals became more and more apparent by the work of Gauß, Dedekind and Artin [11]. In this vein, it was natural to ask whether a formula like (5) holds for the field $\mathbb{Q}$.

In fact, every $n \in \mathbb{Q}^{\times}$is of the form $n= \pm p_{1}^{e_{1}} \cdots p_{r}^{e_{r}}$ with different primes $p_{1}, \ldots, p_{r}$ and integers $e_{i}$. The unit \pm 1 corresponds to the unit $a$ in formula (4). To get a multiplicative analogue of (5), we 
set $e\left(p_{i}\right):=e_{i}$ and $f\left(p_{i}\right):=p_{i}^{-1}$. For the infinite prime, we set $e(\infty):=1$ and $f(\infty):=|n|$. So we get the product formula for $\mathbb{Q}$ :

$$
\prod_{i=1}^{r} f\left(p_{i}\right)^{e\left(p_{i}\right)} \cdot f(\infty)^{e(\infty)}=1 .
$$

An analogous product formula holds for algebraic number fields (see, e. g., [8], III.1.3), or more generally, for global fields [10]. Artin and Whaples [1,2] have shown that the product formula for algebraic number fields follows by formal arguments from its validity for $\mathbb{Q}$, and that a similar statement holds for function fields.

For a global field $K$, (finite or infinite) primes can be conceived as equivalence classes of absolute values $v: K \rightarrow \mathbb{R}$, normalized in a natural way using the modulus function of the locally compact completion $\widehat{K}_{v}$ (see [4], VI.9, Proposition 1). In this way, the product formula admits a natural formulation, but we feel that it remains to be surprising: it just happens to be true - still a mystery! It deeply touches the relationship between the additive and the multiplicative structure of $\mathbb{Q}$.

To reveal this mystery, attempts have been made to elaborate on the concept of "prime" in a field $[6,7,5,13]$. In the present paper, we give a new definition, partly anticipated by Krull [7], which might also shed some more light upon the distinction between finite and infinite primes of an algebraic number field. Namely, we define a prime in a field $K$ to be a subset $P \subset K$ with the following properties:

(P1) $\quad 1 \notin P$.

(P2) $a, b \in P \Rightarrow a b \in P$.

(P3) $a b \in P \Rightarrow a \in P$ or $b \in P$.

(P4) $\exists d \in K^{\times}: P+P \subset d P$.

As a consequence, the subsets $a P$ of $K$ with $a \in K^{\times}$form a chain. There are two types of primes - we call them algebraic and transcendental - such that the algebraic primes correspond to valuation rings in $K$. For every prime $P$, there is a largest algebraic prime $Q \subset P$ (Proposition 7.2); we denote it by $P_{\circ}$. If $P_{\circ}=0$, we call $P$ purely transcendental. For an algebraic number field $K$, the purely transcendental primes just correspond to the infinite primes.

There are unexpected properties of our concept of prime. As is well-known, the valuation rings of a field form a dual tree under inclusion. More generally, we show that all primes of a field $K$ form a tree which is almost self-similar (section 2). Namely, we introduce a binary operation $P * Q$ on the primes which satisfies $P \subset P * Q$ (Proposition 2.4). Thus even for $K=\mathbb{Q}$, there are a lot more primes $P \subset \mathbb{Q}$ than rational prime numbers. A further investigation of this operation might be desirable.

Two primes define the same topology on $K$ if and only if their intersection is non-zero (Proposition 3.3). Up to a constant, we associate a unique pseudo-valuation $v: K \rightarrow[0, \infty]$ to any prime of $K$ (Proposition 3.4). This means that $v(a)=0$ may also occur for non-zero $a \in K$. For a minimal (non-zero) prime $P$, the corresponding pseudo-valuation is of the form $v^{t}$ with $t>0$ for some absolute value $v$, and then $P$ can be recovered from $v$. Every prime $P$ makes $K$ into a separated topological field such that the completion $\widehat{K}_{P}$ is again a topological field. If $P$ is open in its topology, then $P$ is induced by a prime of $\widehat{K}_{P}$ (Theorem 3.1).

We introduce compact primes, which are in a sense totally bounded (Definition 4.1). They always contain a minimal prime which is again compact. For example, all the primes of $\mathbb{Q}$ - not only the minimal ones - are compact. To any compact prime in any field, we associate a Haar integral and 
show that it admits a natural normalization (Theorem 6.1). For the minimal primes of $\mathbb{Q}$ or $\mathbb{F}_{p}[x]$, this normalization corresponds to the standard normalization of absolute values. So the product formula becomes a natural statement on the minimal primes of certain fields.

A special case of our concept of prime already occurs in a paper of W. Krull [7]. Such primes are open in their topology, and we call them Krull primes. We show that every minimal prime is a Krull prime, and that every prime $P$ of a field $K$ contains a largest Krull prime $P^{\circ}$ (Proposition 3.1). There is a close relationship between algebraic primes and Krull primes. Namely, we show that $P_{\circ} \subset P^{\circ}$, so that there is no other prime between $P_{\circ}$ and $P^{\circ}$. Therefore, every compact Krull prime is either algebraic or purely transcendental (Proposition 7.5). Furthermore, a non-zero purely transcendental prime is a Krull prime if and only if it is minimal. The same holds for compact primes (Corollary of Proposition 7.6).

\section{$1 \quad$ Primes}

The two examples of the introduction show that up to units, the elements of certain fields are given by their values at minimal "primes", where a relation between these values is given by a product formula (6). Let us first give a general definition of a prime.

For subsets $P, Q$ of a field $K$ and $c \in K$, define $P+Q:=\{a+b \mid a \in P, b \in Q\}$ and $P Q:=$ $\{a b \mid a \in P, b \in Q\}$. If $P=\{a\}$, we simply write $a+Q:=\{a\}+Q$ and $a Q:=\{a\} Q$. The unit group of a ring $R$ will be denoted by $R^{\times}$.

Definition 1.1. Let $K$ be a field. We call a non-empty subset $P \subset K$ a prime of $K$ if the following are satisfied $(\forall a, b \in K)$ :

$$
\begin{aligned}
& 1 \notin P . \\
& a, b \in P \Rightarrow a b \in P . \\
& a b \in P \Rightarrow a \in P \text { or } b \in P . \\
& \exists d \in K^{\times}: P+P \subset d P .
\end{aligned}
$$

From $(\mathrm{P} 1)$ and $(\mathrm{P} 2)$, we get $-1 \notin P$, since $(-1)(-1)=1$. Therefore, $(-1)(-a)=a$ gives

$$
a \in P \Leftrightarrow-a \in P
$$

by virtue of (P3). By (P4), every $a \in P$ satisfies $0=a-a \in P+P \subset d P$ for some $d \in K^{\times}$. Hence

$$
0 \in P
$$

It is easily checked that $P=0:=\{0\}$ is a prime. We call it the trivial prime. By (P1) and (P2), a finite field has only the trivial prime. For a prime $P$, we call

$$
\widetilde{P}:=\{a \in K \mid a P \subset P\}
$$

the hull and

$$
\partial P:=\widetilde{P} \backslash P
$$

the boundary of $P$. The trivial prime 0 satisfies $\widetilde{0}=K$ and $\partial 0=K^{\times}$. 
Proposition 1.1. Let $K$ be a field with a prime $P$. The $a \widetilde{P}$ with $a \in K^{\times}$form a chain.

Proof. For $a, b \in K^{\times}$, assume that $a \widetilde{P} \not \subset b \widetilde{P}$. Then $a c \notin b \widetilde{P}$ for some $c \in \widetilde{P}$. For every $p \in P$, this gives $a c b^{-1} \cdot b a^{-1} p=c p \in P$, hence $b a^{-1} p \in P$ by (P3). Therefore, $b a^{-1} \in \widetilde{P}$, and thus $b \widetilde{P}=a \cdot b a^{-1} \widetilde{P} \subset a \widetilde{P}$.

Proposition 1.2. Let $K$ be a field with a prime $P$. Then

$$
\partial P=\left\{a \in K^{\times} \mid a P=P\right\}=\left\{a \in K^{\times} \mid a, a^{-1} \notin P\right\} .
$$

Proof. Assume that $a \in \partial P$. Then $a \neq 0$ and $a P \subset P$. For $b \in P$, we have $a \cdot a^{-1} b=b$. Thus (P3) gives $a^{-1} b \in P$. Therefore, $b \in a P$, which yields $P \subset a P \subset P$. Now let $a \in K^{\times}$with $a P=P$ be given. Then $a a^{-1}=1 \notin P$ implies $a^{-1} \notin P$. Similarly, $a^{-1} P=P$, hence $a \notin P$. Thus $\{a \in K \mid a P=P\} \subset\left\{a \in K^{\times} \mid a, a^{-1} \notin P\right\}$. Finally, assume that $a \in K^{\times}$with $a, a^{-1} \notin P$. For $b \in P$, this gives $a^{-1} \cdot a b=b$, hence $a b \in P$ by (P3). Consequently, $a \in \widetilde{P} \backslash P=\partial P$.

Note that (11) implies that $\partial P$ is a subgroup of $K^{\times}$, and

$$
a \notin \widetilde{P} \Leftrightarrow a^{-1} \in P
$$

for all $a \in K^{\times}$, that is, $P$ and $\widetilde{P}$ determine each other. Furthermore, $-1 \in K \backslash P$, and thus $-1 \in \partial P$.

Definition 1.2. We call a prime $P$ of a field $K$ algebraic if $P+P \subset P$, that is, $(\mathrm{P} 4)$ holds for $d=1$, otherwise transcendental.

If $P$ is algebraic, then $(\widetilde{P}+\widetilde{P}) P \subset \widetilde{P} P+\widetilde{P} P \subset P+P \subset P$, which gives $\widetilde{P}+\widetilde{P} \subset \widetilde{P}$. Thus $\widetilde{P}$ is a subring of $K$, and by (12), it follows that $K$ is the quotient field of $\widetilde{P}$. By Proposition 1.1, the principal ideals of $\widetilde{P}$ form a chain. Therefore, all ideals of $\widetilde{P}$ form a chain. Namely, if $I \not \subset J$ are ideals of $\widetilde{P}$, there is some $a \in I \backslash J$. For all $b \in J$, this gives $a \widetilde{P} \not \subset b \widetilde{P}$, hence $b \widetilde{P} \subset a \widetilde{P} \subset I$. Furthermore, $P$ is an ideal of $\widetilde{P}$, and by $(11)$ and $(12), \partial P=(\widetilde{P})^{\times}$. Thus $P$ is the unique maximal ideal of $\widetilde{P}$. In other words, $\widetilde{P}$ is a valuation domain.

Example. For $K=\mathbb{Q}$, every rational prime $p$ gives rise to an algebraic prime

$$
P_{p}=\left\{\frac{p a}{b} \in \mathbb{Q} \mid a, b \in \mathbb{Z}, p \nmid b\right\} .
$$

By (12), we get $\widetilde{P_{p}}=\mathbb{Z}_{p}$, whence

$$
\widetilde{P_{p}} / P_{p}=\mathbb{Z}_{p} / p \mathbb{Z}_{p} \cong \mathbb{Z} / p \mathbb{Z}
$$

Moreover, $\mathbb{Q}$ has a transcendental prime

$$
P_{\infty}:=(-1,1) \cap \mathbb{Q}=\{a \in \mathbb{Q}|| a \mid<1\} .
$$

Here (P4) holds for $d=2$.

\section{The primes of $\mathbb{Q}$}

By (12), two primes $P \supset Q$ of a field $K$ satisfy

$$
Q \subset P \subset \widetilde{P} \subset \widetilde{Q},
$$

hence $c P \subset c \widetilde{Q} \subset Q$ for all $c \in Q$. More precisely: 
Proposition 2.1. Let $K$ be a field with a prime P. For any submonoid $U$ of $K^{\times}$with $\widetilde{P} \subset U$,

$$
Q:=\{0\} \cup\left\{a \in K^{\times} \mid a^{-1} \notin U\right\}
$$

is a prime with $Q \subset P$ and $\widetilde{Q}=U$. Every prime $Q \subset P$ is of this form.

Proof. Let $U$ be a submonoid of $K^{\times}$, and $\widetilde{P} \subset U$. By (12), this implies that $Q \subset P$. Assume that $a, b \in Q \cap K^{\times}$. Then $a^{-1}, b^{-1} \notin U$. So $b^{-1} \notin \widetilde{P}$, and therefore, $b \in P \subset U$. Now $a b \notin Q$ would yield $a^{-1} b^{-1} \in U$, whence $a^{-1}=a^{-1} b^{-1} \cdot b \in U$, which is impossible! Thus (P1) and (P2) hold for $Q$.

To prove (P3), we have to verify the implication $a, b \notin Q \Rightarrow a b \notin Q$, that is, $a^{-1}, b^{-1} \in U \Rightarrow$ $a^{-1} b^{-1} \in U$. This follows since $U$ is multiplicatively closed.

Finally, $Q+Q \subset P+P \subset d P$ holds for some $d \in K^{\times}$. To prove (P4), we can assume that $Q \neq 0$. For arbitrary $a \in K \backslash U$ and $b \in P \cap K^{\times}$, we have $a \notin \widetilde{P}$. Thus $a^{-1} \in P$. So $b^{-1} a \in U$ would give $a \in b U \subset U$, a contradiction. Hence $b^{-1} a \notin U$, and thus $b a^{-1} \in Q$. So we get $P \subset a Q$, i. e. $Q+Q \subset d a Q$, which completes the proof of (P4).

Conversely, let $Q \subset P$ be a prime. Then (12) shows that $Q$ is of the form (17) with a submonoid $U:=\widetilde{Q}$ of $K^{\times}$.

Corollary. For each pair $P, Q$ of primes of a field $K$, there is an infimum $P \wedge Q$.

Proof. By Proposition 2.1, the submonoid $\widetilde{P} \widetilde{Q}$ corresponds to a prime $P \wedge Q$ with the desired property.

Definition 2.1. We say that two primes $P$ and $Q$ of a field $K$ are dependent if $P \wedge Q \neq 0$, otherwise independent. The minimal among the non-zero primes are called minimal primes.

Minimal primes can be characterized as follows.

Proposition 2.2. A non-trivial prime $P$ of a field $K$ is minimal if and only if for every pair $a, b \in$ $P \cap K^{\times}$, there exists some $n \in \mathbb{N}$ with $a^{n} \in b P$.

Proof. Assume that $a, b \in P \cap K^{\times}$. Then $U:=\bigcup_{n \in \mathbb{N}} a^{-n} P$ is a submonoid of $K^{\times}$, and $\widetilde{P} \subset$ $a^{-1} P \subsetneq U$. Suppose that $a^{n} \notin b P$ for all $n \in \mathbb{N}$. Then $b^{-1} \notin U$. By Proposition 2.1, there is a non-trivial prime $Q \subsetneq P$ with $\widetilde{Q}=U$.

Conversely, let $Q$ be a prime with $0 \neq Q \subsetneq P$. Choose $a \in P \backslash Q$ and $b \in Q \cap K^{\times}$. Then $a \notin Q$ implies that $a^{-1} \in \widetilde{Q}$, hence $a^{-n} \in \widetilde{Q}$ for all $n \in \mathbb{N}$. Furthermore, $b P \subset b \widetilde{P} \subset b \widetilde{Q} \subset Q$, and thus $a^{-n} b P \subset a^{-n} Q \subset Q \subset P$. Hence $a^{-n} b \in \widetilde{P}$, that is, $a^{n} b^{-1} \notin P$ for all $n$. Thus $a^{n} \notin b P$ holds for $n \in \mathbb{N}$.

Now we are able to give an explicit description of the minimal primes of $\mathbb{Q}$. To this end, we need two auxiliary results.

Lemma 2.1. Let $P$ be a prime of $\mathbb{Q}$, such that $P+P \subset d P$ for some $d \in \mathbb{Q}^{\times}$. Then $n P \subset d^{k} P$ holds for all $k \in \mathbb{N}$ and $n \in \mathbb{Z}$ with $|n| \leqslant 2^{k}$.

Proof. For $k=0$, this is trivial. If the assertion holds for some $k \in \mathbb{N}$, then every $n \in \mathbb{Z}$ with $|n| \leqslant 2^{k+1}$ can be written as a sum $n=r+s$ in $\mathbb{Z}$ such that $|r|,|s| \leqslant 2^{k}$. Hence $n P \subset r P+s P \subset$ $d^{k} P+d^{k} P \subset d^{k+1} P$. 
Lemma 2.2. Let $P$ be a prime of $\mathbb{Q}$, and let $a>b$ be relatively prime in $\mathbb{N}$, such that $a(P+P) \subset b P$. Then

$$
n a^{a} P \subset b^{a+k-1} P
$$

holds for all $k \in \mathbb{N}$ and $n \in \mathbb{Z}$ with $|n|<a^{k}$.

Proof. We proceed by induction. For $k=0$, the statement (18) is trivial. Suppose that (18) is shown for some $k \in \mathbb{N}$. We prove (18) for $k+1$. Assume that $|n|<a^{k+1}$. Since $a$ and $b$ are relatively prime, there are $m, r \in \mathbb{Z}$ with

$$
n=m a+r b^{k}
$$

If $r$ is replaced by $r+a$, the equation holds for $m-b^{k}$ instead of $m$. So we can assume that $|r|<a$. For $r \neq 0$, there are still two possibilities. We choose $r$ such that the signs of $n$ and $r$ are not different. Then $|m|=\frac{1}{a}\left|n-r b^{k}\right|<a^{k}$. So the inductive hypothesis gives $m a^{a} P \subset b^{a+k-1} P$, hence $n a^{a} P \subset m a \cdot a^{a} P+r b^{k} \cdot a^{a} P \subset b^{a+k-1} a P+r b^{k} a^{a} P$. Since $|r| \leqslant a-1<2^{a-1}$, Lemma 2.1 implies that $r P \subset\left(\frac{b}{a}\right)^{a-1} P$. Therefore, we get $n a^{a} P \subset b^{a+k-1} a P+b^{a+k-1} a P \subset b^{a+k-1} b P=b^{a+k} P$.

As a consequence of Lemma 2.2, we have

Lemma 2.3. Let $P$ be a prime of $\mathbb{Q}$, and assume that $1<c \in P$. Then there exists an integer $e>1$ with $\mathbb{Z} e \subset P$ and $e(P+P) \subset P$.

Proof. By (P4), there is some $d \in \mathbb{Q}^{\times}$with $P+P \subset d P$. Choose $i \in \mathbb{N}$ such that $c^{i}>|d|$. Then $c^{i} \cdot|d|^{-1}=\frac{a}{b}$ with relatively prime $a, b \in \mathbb{N}$. Thus $a>b$ and $a(P+P)=b c^{i} d^{-1}(P+P) \subset b c^{i} P \subset b P$. For every $m \in \mathbb{Z}$, there exists some $k \in \mathbb{N}$ with $|m| \cdot b^{a}<\left(\frac{a}{b}\right)^{k}$. We set $n:=m \cdot b^{a+k}$. Then $|n|=|m| \cdot b^{a+k}<a^{k}$. By Lemma 2.2, this implies that $n a^{a} P \subset b^{a+k-1} P$, that is, $m a^{a} b P \subset P$ for all $m \in \mathbb{Z}$, and thus $\mathbb{Z} a^{a+1}=\mathbb{Z} a^{a} b \cdot \frac{a}{b} \subset P$. Furthermore, $a^{a+1}(P+P)=a^{a} \cdot a(P+P) \subset a^{a} b P \subset P$.

Now we show that the list of minimal primes of $\mathbb{Q}$ given in (13) and (15) is complete. The first part of the following theorem is essentially due to Ostrowski [9] (cf. [12]).

Theorem 2.1. Every minimal prime $P$ of $\mathbb{Q}$ is of the form $P=P_{p}$ with a rational prime $p$ or $p=\infty$, and every prime of $\mathbb{Q}$ contains a minimal prime.

Proof. By Proposition 2.2, the prime $P_{\infty}$ is minimal. Therefore, if $|c|<1$ for all $c \in P$, then $P \subset P_{\infty}$, and thus $P=P_{\infty}$. Otherwise, there exists some $c \in P$ with $c>1$. By Lemma 2.3, there is an integer $e>1$ with $\mathbb{Z} e \subset P$ and $e(P+P) \subset P$. Assume that $e=p_{1} \cdots p_{r}$ with rational primes $p_{i}$. Suppose that $e p_{i}^{-r} \in P$ holds for all $i$. Then $1=\left(e p_{1}^{-r}\right) \cdots\left(e p_{r}^{-r}\right) \in P$, a contradiction. Hence $e p^{-r} \notin P$ for some prime $p \mid e$. This implies that $e^{-1} p^{r} \in \widetilde{P}$. For every $n \in \mathbb{Z}$, we thus get $(n p)^{r}=n^{r} e \cdot e^{-1} p^{r} \in P \widetilde{P} \subset P$. Therefore, $n p \in P$, and thus $\mathbb{Z} p \subset P$.

Now let $p \nmid n$ for some $n \in \mathbb{Z}$. Then there are $u, v \in \mathbb{Z}$ with $u n+v p^{r+1}=1$. So the residue class $n+p^{r+1} \mathbb{Z}$ is invertible in $\mathbb{Z} / p^{r+1} \mathbb{Z}$. Therefore, we find some $k \in \mathbb{N} \backslash 0$ with $n^{k} \equiv 1\left(p^{r+1}\right)$. With $s:=(r+1) k$, we also have $n^{s} \equiv 1\left(p^{r+1}\right)$, and thus $1=n^{s}+m p^{r+1}$ for some $m \in \mathbb{Z}$. Suppose that $n^{s} \in p^{r} P$. Then $1 \in p^{r} P+p^{r} P=e^{-1} p^{r} \cdot e(P+P) \subset \widetilde{P} P \subset P$, a contradiction. Hence $n^{s} p^{-r} \notin P$, and thus $n^{-s} p^{r} \in \widetilde{P}$. So we get $\left(n^{-1} p\right)^{s}=n^{-s} p^{(r+1) k} \in n^{-s} p^{r} P \subset P$. This gives $n^{-1} p \in P$, whence $P_{p} \subset P$.

Remark. There exist non-minimal primes in $\mathbb{Q}$. For example,

$$
P:=2 \mathbb{Z}_{2} \cup\left(\mathbb{Z}_{2} \cap 3 \mathbb{Z}_{3}\right)
$$


is a prime. In fact, this is a general phenomenon. We show first that the primes form a tree with the trivial prime as a root:

Proposition 2.3. Let $K$ be a field with a prime $P$. Then the primes $Q \subset P$ form a chain.

Proof. Let $Q, Q^{\prime}$ be primes with $Q, Q^{\prime} \subset P$ and $Q^{\prime} \not \subset Q$. Then there is an element $a \in Q^{\prime} \backslash Q$. For $b \in Q$, the inclusions (16) give $b \widetilde{P} \subset b \widetilde{Q} \subset Q$. Hence $a \widetilde{P} \not \subset b \widetilde{P}$. Therefore, Proposition 1.1 yields $b \in b \widetilde{P} \subset a \widetilde{P} \subset a \widetilde{Q^{\prime}} \subset Q^{\prime}$, and thus $Q \subset Q^{\prime}$.

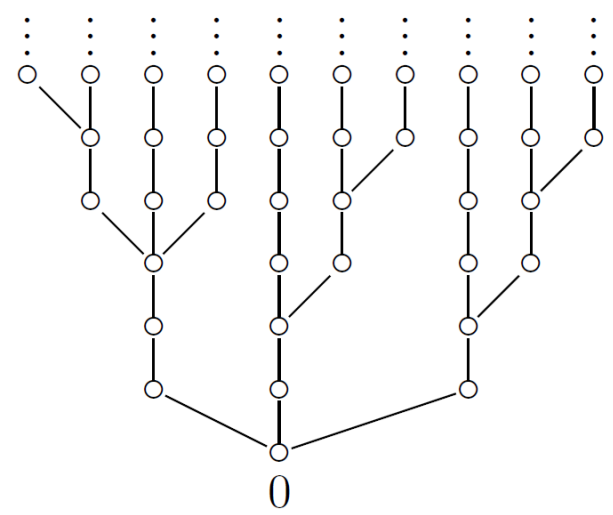

Moreover, the tree of primes has a strong symmetry:

Proposition 2.4. Let $K$ be a field with two primes $P$ and $Q$. Then

$$
P * Q:=P \cup(\widetilde{P} \cap Q)
$$

is a prime of $K$.

Proof. Obviously, $1 \notin P * Q$. Assume that $a, b \in P * Q$. If $a \in P$ or $b \in P$, then $a b \in P \subset P * Q$ since $a, b \in \widetilde{P}$. For $a, b \in \widetilde{P} \cap Q$, however, we have $a b \in \widetilde{P} \cap Q$. This proves (P2). To verify (P3), let $a, b \in K$ with $a b \in P * Q$ be given. If $a b \in P$, then $a \in P$ or $b \in P$. Therefore, assume that $a b \in \widetilde{P} \cap Q$ and $a, b \notin P$. Then $a^{-1}, b^{-1} \in \widetilde{P}$. Hence $a=a b \cdot b^{-1} \in \widetilde{P}$, and similarly, $b \in \widetilde{P}$. Therefore, $a \in \widetilde{P} \cap Q$ or $b \in \widetilde{P} \cap Q$. Finally, assume that $d \in K^{\times}$with $P+P \subset d P$. If $P=0$, we have $P * Q=Q$. Otherwise, there exists some $a \in P \backslash 0$. Now $(\widetilde{P}+\widetilde{P}) P \subset P+P \subset d P$, hence $(\widetilde{P}+\widetilde{P}) d^{-1} \subset \widetilde{P}$. Consequently, $(P * Q)+(P * Q) \subset \widetilde{P}+\widetilde{P} \subset d \widetilde{P}=d a^{-1} a \widetilde{P} \subset d a^{-1}(P * Q)$.

We call $P * Q$ the lexicographic product of $P$ and $Q$. Thus for a fixed $P$, every prime $Q$ yields a prime $P * Q \supset P$ which may coincide with $P$. Apart from this exception, the tree of primes is self-similar, i. e. the whole tree sits above each prime.

\section{The topology of a prime}

Let $K$ be a field with a prime $P$. For $a, b \in K$, we define

$$
a \leqslant b: \Leftrightarrow a \widetilde{P} \subset b \widetilde{P}
$$


and $a<b$ if $a \ngtr b$. Then

$$
\begin{aligned}
a \leqslant b \leqslant c & \Rightarrow a \leqslant c \\
a \leqslant b & \Rightarrow a c \leqslant b c
\end{aligned}
$$

holds for $a, b, c \in K$, and Proposition 1.1 yields

$$
a \leqslant b \text { oder } b \leqslant a \text {. }
$$

In particular, this implies the reflexity $a \leqslant a$ for all $a \in K$. By (11), we have $a P=b P \Leftrightarrow b^{-1} a P=$ $P \Leftrightarrow b^{-1} a \in \partial P$ for $a, b \in K^{\times}$. Therefore, (19) induces a linear order on $K^{\times} / \partial P$. From (19) and (12), we get

$$
\widetilde{P}=\{a \in K \mid a \leqslant 1\} ; \quad P=\{a \in K \mid a<1\} .
$$

For $a \in K$ and $c \in K^{\times}$, we define the $c$-neighbourhood of $a$ as follows:

$$
U_{c}(a)=U_{c}^{P}(a):=a+c P .
$$

A subset $U \subset K$ is open with respect to $P$ if for every $a \in U$, there is an element $c \in K^{\times}$with $U_{c}(a) \subset U$. The $P$-open sets define a topology on $K$, but it is not clear in advance that the $c$ neighbourhoods (23) are neighbourhoods in this topology. The following proposition shows that this is in fact the case.

Proposition 3.1. Let $K$ be a field with a prime $P$. The set

$$
P^{\circ}:=\left\{a \in P \mid \exists n \in \mathbb{N}: a^{n}(P+P) \subset P\right\}
$$

is open. If $P$ is minimal, then $P^{\circ}=P$.

Proof. Assume that $a \in P^{\circ}$, say, $a^{n}(P+P) \subset P$. Then $a^{2 n}(P+P+P+P) \subset a^{n}(P+P) \subset P$, and by induction, we get

$$
a^{m n}(P+\cdots+P) \subset P
$$

for $m \in \mathbb{N}$, where $P+\cdots+P$ may have at most $2^{m}$ summands. We set $b:=a^{2 n(n+1)}$. Now let $p \in P$ be arbitrary. Expanding $(a+b p)^{2 n}$ gives $2^{2 n}$ summands, namely, $a^{2 n}$ plus $2^{2 n}-1$ summands in $b P$. So we get

$$
\begin{aligned}
(a+b p)^{2 n}(P+P) & \subset\left(a^{2 n}+b a^{-2 n^{2}} P\right)(P+P) \subset\left(a^{2 n}+a^{2 n} P\right)(P+P) \\
& \subset a^{2 n}(P+P)+a^{2 n}(P+P) \subset a^{n} P+a^{n} P \subset P .
\end{aligned}
$$

This yields $a+b P \subset P^{\circ}$. Finally, let $P$ be minimal and $d \in K^{\times}$with $P+P \subset d P$. Then $P \subset d P$, hence $d^{-1} \in \widetilde{P}$. For $a \in P$, Proposition 2.2 yields an $n \in \mathbb{N}$ with $a^{n} \in a d^{-1} P$, whence $a^{n}(P+P) \subset a d^{-1}(P+P) \subset a P \subset P$. Thus $a \in P^{\circ}$.

In section 7 , we will show that $P^{\circ}$ is again a prime.

Corollary. Let $K$ be a field with a minimal prime $P$. For every $a \in P$, there are $b, c \in P \cap K^{\times}$such that $a \widetilde{P}+b P \subset c \widetilde{P}$.

Proof. Assume that $P+P \subset d P$ with $d \in K^{\times}$and $a \in P$. By Proposition 3.1, there is some $b \in d^{-1} P \cap K^{\times}$with $a+b d P \subset P$. We show first that $a \widetilde{P}+b P \subset P$. To this end, assume that $e \in \widetilde{P}$. 
Case I: $e \in d^{-1} P$. Then $a e+b P \subset d^{-1} P+d^{-1} P \subset P$.

Case II: $e \notin d^{-1} P$. Then $d e \notin P$, hence $d^{-1} e^{-1} \in \widetilde{P}$. This gives $a e+b P=e\left(a+b d d^{-1} e^{-1} P\right) \subset$ $e(a+b d P) \subset P$.

If $a \widetilde{P}=P$, we can set $c:=a$. Otherwise, there exists some $c \in P \backslash a \widetilde{P}$. Then $c a^{-1} \notin \widetilde{P}$, hence $c^{-1} a \in P$. By the above, we find an element $b \in d^{-1} P \cap K^{\times} \subset P \cap K^{\times}$with $c^{-1} a \widetilde{P}+b P \subset P$. Whence $a \widetilde{P}+c b P \subset c P \subset c \widetilde{P}$.

Lemma 3.1. Let $K$ be a field with a prime $P$. Assume that $d \in K^{\times}$with $P+P \subset d P$. Then every $a, b \in K^{\times}$with $b \in a d^{-1} P$ satisfies

$$
\forall p \in P:(a+b p)^{-1}-a^{-1} \in d a^{-2} b P .
$$

Proof. Assume that $p \in P$. Then $b p \in a P$ implies that $a+b p \neq 0$. Suppose that $\left(1+a^{-1} b p\right) d \in P$. Then $d=\left(1+a^{-1} b p\right) d-a^{-1} b p d \in P+P \subset d P$, a contradiction. Hence $\left(1+a^{-1} b p\right)^{-1} d^{-1} \in \widetilde{P}$. From $\left(1+a^{-1} b p\right)^{-1}\left(1+a^{-1} b p\right)=1$ we get

$$
\left(1+a^{-1} b p\right)^{-1}+\left(1+a^{-1} b p\right)^{-1} a^{-1} b p=1 .
$$

Multiplying by $a^{-1}$ gives $(a+b p)^{-1}+\left(1+a^{-1} b p\right)^{-1} a^{-2} b p=a^{-1}$, i. e. $(a+b p)^{-1}-a^{-1}=-(1+$ $\left.a^{-1} b p\right)^{-1} a^{-2} b p \in d \widetilde{P} \cdot a^{-2} b P \subset d a^{-2} b P$.

Theorem 3.1. Let $K$ be a field with a prime $P$. The topology of $P$ makes $K$ into a separated topological field, and the completion $\widehat{K}_{P}$ of $K$ with respect to $P$ is a topological field. If $P$ is open, then

$$
\widehat{P}:=\bigcup_{a \in P} \overline{a \widetilde{P}}
$$

is a prime in $\widehat{K}_{P}$ with $\widehat{P} \cap K=P$, and $\widehat{P}$ defines the topology of $\widehat{K}_{P}$.

Proof. By (P4), there is some $d \in K$ with $P+P \subset d P$. This implies that $K$ is a topological ring. Hence $K$ is a topological field by Lemma 3.1.

To show that the same holds for the completion $\widehat{K}$ of $K$, we have to verify that the image under the mapping $x \mapsto x^{-1}$ of every Cauchy filter $\mathfrak{C}$ in $K$ which does not have a cluster point at 0 , is a Cauchy filter (see [3], III.6.8, Proposition 7). Thus let $c \in K^{\times}$and $C \in \mathfrak{C}$ be such that $C \cap c P=\varnothing$. We can assume that $a-b \in c d^{-1} P$ for all $a, b \in C$. Choose any $a \in C$. Suppose that $b \in C \cap c d^{-1} P$. Then $a=(a-b)+b \in c d^{-1} P+c d^{-1} P \subset c P$, a contradiction. Hence $C \cap c d^{-1} P=\varnothing$. This gives $a c^{-1} d \notin P$, and thus $a^{-1} c d^{-1} \in \widetilde{P}$, i. e. $a^{-1} \in c^{-1} d \widetilde{P}$. For an arbitrary $e \in K^{\times}$, we find some $C^{\prime} \subset C$ in $\mathfrak{C}$ with $a-b \in c^{2} d^{-2} e P$ for all $a, b \in C^{\prime}$. This gives $a^{-1}-b^{-1}=a^{-1} b^{-1}(b-a) \in c^{-2} d^{2} \widetilde{P} \cdot c^{2} d^{-2} e P \subset e P$ for $a, b \in C^{\prime}$.

Now let $P$ be open. We show first that for $a \in P$,

$$
\overline{a \widetilde{P}} \subset P
$$

holds in $K$. For $a=0$, this is trivial. So we assume that $a \in P \cap K^{\times}$. Let $b \notin P$ be given. Then $b^{-1} \in \widetilde{P}$, which gives $\left(a^{-1} b\right)^{-1}=b^{-1} a \in P$. Since $P$ is open, the continuity of $x \mapsto x^{-1}$ implies that $\left(a^{-1} b+c P\right)^{-1} \subset P$ for some $c \in K^{\times}$. Hence $\left(a^{-1} b+c P\right) \cap \widetilde{P}=\varnothing$, and thus $(b+a c P) \cap a \widetilde{P}=\varnothing$. This proves (26). Together with (25), we infer that $\widehat{P} \cap K=P$. 
Furthermore, (26) shows that $\widehat{K}_{P}$ satisfies (P1). If $b, c \in \widehat{P}$, then $b, c \in \overline{a \widetilde{P}}$ for some $a \in P$. Hence $b c \in \overline{a \widetilde{P}} \cdot \overline{a \widetilde{P}} \subset \overline{a^{2} \widetilde{P}} \subset \widehat{P}$, which gives (P2). Similarly, (P4) is easily verified.

To prove (P3), assume that $b, c \in \widehat{K}_{P}$ satisfy $b c \in \widehat{P}$ and $b \notin \widehat{P}$, say, $b c \in \overline{a \widetilde{P}}$ for some $a \in P$. Then $b \notin \overline{p \widetilde{P}}$ for all $p \in P$. For any $p \in P$, we find a neighbourhood $U$ of 0 in $\widehat{K}_{P}$ such that $(b+U) \cap p \widetilde{P}=\varnothing$. Hence $(b c+U c) \cap p c \widetilde{P}=\varnothing$. On the other hand, $(b c+U c) \cap a \widetilde{P} \neq \varnothing$. Thus for any $p \in P$, we get $a \widetilde{P} \not \subset p c \widetilde{P}$. If $p \neq 0$, this implies that $p^{-1} c^{-1} a \notin \widetilde{P}$. Hence $p c a^{-1} \in P$ for all $p \in P$. Consequently, $c a^{-1} \in \widetilde{P}$, and thus $c \in a \widetilde{P} \subset \widehat{P}$.

It remains to verify that $\widehat{P}$ defines the topology of $\widehat{K}_{P}$. For $a \in P$, we have $\overline{a P} \subset \widehat{P}$. Conversely, (26) implies that $\widehat{P} \subset \bar{P}$. This completes the proof.

In particular, Theorem 3.1 implies that the limit of a sequence in $K$ with respect to a prime $P$ is unique. A minimal prime $P$ is uniquely determined by its topology:

Proposition 3.2. Let $K$ be a field with a minimal prime $P$. Then

$$
P=\left\{a \in K \mid \lim _{n \rightarrow \infty} a^{n}=0\right\} .
$$

Proof. Let $a \in P$. By Proposition 2.2, every $c \in K^{\times}$gives rise to some $N \in \mathbb{N}$ with $a^{N} \in c P$. Therefore, $a^{n} \in c P$ for all $n \geqslant N$, and thus $\lim _{n \rightarrow \infty} a^{n}=0$. On the other hand, if $a \notin P$, then $a^{-1} \in \widetilde{P}$, which gives $a^{-n} \in \widetilde{P}$ for all $n \in \mathbb{N}$. But this implies that $a^{n} \notin P$ for all $n$.

If a prime contains a minimal prime $P$, then $P$ can be determined topologically by means of Proposition 3.2. The following proposition decides whether two primes define the same topology.

Proposition 3.3. For two primes $P, Q \neq 0$ of a field $K$, the following are equivalent.

(a) $P Q \neq K$.

(b) $\widetilde{P} \widetilde{Q} \neq K$.

(c) $P$ and $Q$ are dependent (see Definition 2.1).

(d) There is some $a \in K^{\times}$with $a P \subset Q$.

(e) $P$ and $Q$ define the same topology.

Proof. (a) $\Rightarrow$ (b): Suppose that $\widetilde{P} \widetilde{Q}=K$. For $a \in P \cap K^{\times}$and $b \in Q \cap K^{\times}$, this implies that $K=a b \widetilde{P} \widetilde{Q} \subset P Q$, which is impossible. The implication (b) $\Rightarrow$ (c) follows by Proposition 2.1.

(c) $\Rightarrow$ (d): For every $a \in(P \wedge Q) \cap K^{\times}$, we have $a P \subset P \wedge Q \subset Q$.

(d) $\Rightarrow$ (a): From $a P \subset Q$ with $a \in K^{\times}$, we get $a P Q \subset Q^{2} \subset Q$, hence $P Q \subset a^{-1} Q \neq K$. Therefore, condition (d) is symmetric. It states that every $Q$-open set with respect to $P$ is open. Hence $(\mathrm{d}) \Leftrightarrow(\mathrm{e})$.

Let $K$ be a field with a prime $P$. A function $f: K \rightarrow[0, \infty]$ is said to be monotonous (with respect to $P$ ) if

$$
a \leqslant b \Rightarrow f(a) \leqslant f(b)
$$

holds for all $a, b \in K$. We call $f$ strictly monotonous if (28) can be replaced by an equivalence. For the following definition, we set

$$
\infty \cdot a=a \cdot \infty:=\infty
$$

for $0<a \leqslant \infty$. 
Definition 3.1. Let $K$ be a field with a prime $P$. We call $v: K \rightarrow[0, \infty]$ a pseudo-valuation with respect to $P$ if $v$ is monotonous with $v(0)=0$ and $v(1)=1$, such that

$$
v(a b)=v(a) v(b)
$$

holds for all $a, b \in K$ with $v(a) \notin\{0, \infty\}$. We call $v$ finite if $v(a)<\infty$ for all $a \in K$. If $v$ is finite and strictly monotonous, we say that $v$ is a P-valuation.

For $a \in K^{\times}$, the definition yields

$$
v(a)=0 \Leftrightarrow v\left(a^{-1}\right)=\infty .
$$

Note that by (22), the prime $P$ of a $P$-valuation $v$ is given by $P=\{a \in K \mid v(a)<1\}$. By [4], VI.6.1, a $P$-valuation is of the form $v^{t}$, where $t>0$ and $v$ is an absolute value on $K$.

Proposition 3.4. Let $K$ be a field with a prime $P$. For every $c \in K \backslash \widetilde{P}$ and every real number $r>1$, there is exactly one pseudo-valuation $v: K \rightarrow[0, \infty]$ with $v(c)=r$. If $P$ is minimal, then $v$ is a P-valuation.

Proof. First, let $v$ be a pseudo-valuation with $v(c)=r$, and let $a \in K$ be given. If $a \leqslant c^{n}$ for all $n \in \mathbb{Z}$, then $v(a) \leqslant r^{n}$ for all $n$, and thus $v(a)=0$. If, however, $a \geqslant c^{n}$ for all $n \in \mathbb{Z}$, we get $v(a)=\infty$. Otherwise, there exists some $n \in \mathbb{N}$ with $c^{-n} \leqslant a \leqslant c^{n}$, and then $r^{-n} \leqslant v(a) \leqslant r^{n}$, hence $v(a) \notin\{0, \infty\}$. For such an $a$ and an integer $n>0$, there exists some $m \in \mathbb{Z}$ with $c^{m-1} \leqslant a^{n} \leqslant c^{m}$, which gives $r^{m-1} \leqslant v(a)^{n} \leqslant r^{m}$, i. e.

$$
r^{\frac{m-1}{n}} \leqslant v(a) \leqslant r^{\frac{m}{n}}
$$

Thus $v$ is unique:

$$
v(a)=\inf \left\{r^{m / n} \mid m, n \in \mathbb{Z}, n>0, a^{n} \leqslant c^{m}\right\} .
$$

This formula extends to $v(a) \in\{0, \infty\}$. Conversely, we show that (32) defines a pseudo-valuation. Again, $v(a)=0$ respectively $v(a)=\infty$ holds if $a \leqslant c^{n}$ resp. $a \geqslant c^{n}$ for all $n \in \mathbb{Z}$. Therefore, assume that $v(a) \notin\{0, \infty\}$. Then for every $n>0$ in $\mathbb{N}$, there is an integer $m$ with $c^{m-1} \leqslant a^{n} \leqslant c^{m}$. This implies (30) for $v(b) \in\{0, \infty\}$. Assume that $c^{k-1} \leqslant b^{n} \leqslant c^{k}$. Then we get $r^{\frac{m-1}{n}} \leqslant v(a) \leqslant r^{m / n}$ and $r^{\frac{k-1}{n}} \leqslant v(b) \leqslant r^{k / n}$. Hence $r^{\frac{m+k-2}{n}} \leqslant v(a) v(b) \leqslant r^{\frac{m+k}{n}}$. On the other hand, $c^{k+m-2} \leqslant(a b)^{n} \leqslant c^{m+k}$, and thus $r^{\frac{m+k-2}{n}} \leqslant v(a b) \leqslant r^{\frac{m+k}{n}}$. For $n \rightarrow \infty$, this gives (30). Furthermore, (32) implies that $v$ is monotonous, and $v(0)=0, v(1)=1$ holds together with $v(c)=r$. Hence $v$ is a pseudo-valuation. If $P$ is minimal, Proposition 2.2 shows that for every $a \in P$, the inequality $v(a)<1$ holds. Hence $v$ is a $P$-valuation.

Examples. 1. For the transcendental prime $P_{\infty}=(-1,1) \cap \mathbb{Q}$ of $\mathbb{Q}$, the unique $P_{\infty}$-valuation $v: \mathbb{Q} \rightarrow[0, \infty]$ with $r=c>1$ (Proposition 3.4) is given by $v(a):=|a|$. So the completion is $\mathbb{R}$, and $\widehat{P_{\infty}}=(-1,1)$. For a prime $p$ and $P:=p \mathbb{Z}_{p}$, every $a \in \mathbb{Q}^{\times}$admits a unique representation $a=p^{n} \cdot \frac{b}{c}$ with $p \nmid b, c$ and $n \in \mathbb{Z}$. Then $v(a)=|a|_{p}:=p^{-n}$ with $|0|_{p}:=0$ is a $P$-valuation $v: \mathbb{Q} \rightarrow[0, \infty]$, the $p$-adic absolute value of $\mathbb{Q}$. The $c$-neighbourhoods (23) of 0 are then of the form $p^{n} \mathbb{Z}_{p}, n \in \mathbb{Z}$. As usual, we write $\widehat{\mathbb{Q}}_{p}$ for the completion of $\mathbb{Q}$ with respect to $P=p \mathbb{Z}_{p}$. Here we have $\widehat{P}=p \widehat{\mathbb{Z}}_{p}$, where $\widehat{\mathbb{Z}}_{p}$ denotes the ring of $p$-adic numbers.

2. For the field $\mathbb{C}$ of complex numbers, $P:=\{a \in \mathbb{C}|| a \mid<1\}$ is a prime, and the absolute value is a $P$-valuation $v: \mathbb{C} \rightarrow[0, \infty]$. Here we have $\widehat{\mathbb{C}}_{P}=\mathbb{C}$. 


\section{Compact primes and the Haar integral}

In the sequel, we deal with primes like those occuring in algebraic number fields.

Definition 4.1. We call a prime $P$ of a field $K$ compact if for every $b \in K^{\times}$there exist $a_{1}, \ldots, a_{n} \in K$ with

$$
P \subset\left(a_{1}+b P\right) \cup \cdots \cup\left(a_{n}+b P\right)
$$

Example. The primes of $\mathbb{Q}$ are compact: For the prime $P_{\infty}$ this is trivial; for a rational prime $p$, we have $p \mathbb{Z}_{p}=\bigcup_{i=1}^{p^{n-1}} i p+p^{n} \mathbb{Z}_{p}$. So the minimal primes of $\mathbb{Q}$ are compact.

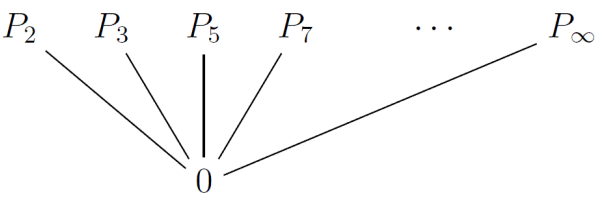

By the following result, the other primes are compact, too:

Proposition 4.1. Let $K$ be a field. The primes $Q$ which are dependent of a non-trivial compact prime $P$ are compact.

Proof. Assume that $b \in K^{\times}$. Since $P \wedge Q \neq 0$, there exists some $a \in(P \wedge Q) \cap K^{\times}$. As $P$ is compact, there are $a_{1}, \ldots, a_{n} \in K$ with $P \subset\left(a_{1}+a^{2} b P\right) \cup \cdots \cup\left(a_{n}+a^{2} b P\right)$. Hence $a Q \subset P \subset$ $\bigcup_{i=1}^{n}\left(a_{i}+a^{2} b P\right) \subset \bigcup_{i=1}^{n}\left(a_{i}+a b Q\right)$, and thus $Q \subset \bigcup_{i=1}^{n}\left(a^{-1} a_{i}+b Q\right)$.

Every compact prime $P$ of a field $K$ leads to a concept of integral. Let $\mathcal{C}_{P}(K)$ denote the set of real functions $f: K \rightarrow \mathbb{R}$ such that $f$ vanishes on $K \backslash c P$ for some $c \in K^{\times}$, and is uniformly continuous with respect to $P$, i. e. for every $\varepsilon>0$ there exists some $b \in K^{\times}$such that $\left|f(a)-f\left(a^{\prime}\right)\right|<\varepsilon$ holds for all $a, a^{\prime} \in K$ with $a-a^{\prime} \in b P$. Clearly, $\mathcal{C}_{P}(K)$ is an $\mathbb{R}$-vector space. We write $f \leqslant g$ for $f, g \in \mathcal{C}_{P}(K)$ if $f(a) \leqslant g(a)$ holds for all $a \in K$. If, in addition, $f \neq g$, we write $f<g$. The subset of the $f>0$ in $\mathcal{C}_{P}(K)$ will be denoted by $\mathcal{C}_{P}^{+}(K)$. We show first that $\mathcal{C}_{P}^{+}(K) \neq \varnothing$.

Proposition 4.2. Let $K$ be a field with a prime $P$. For every $b \in K^{\times}$, there exists a function $f \in \mathcal{C}_{P}^{+}(K)$ which satisfies $f(a)=1$ for all $a \in b P$.

Proof. Since $a \in b P \Leftrightarrow a b^{-1} \in P$, we can assume that $b=1$. Furthermore, we can assume that $P \neq 0$. By (P4), there is an element $d \in K \backslash \widetilde{P}$ with $P+P \subset d P$, and by Proposition 3.4, there exists a pseudo-valuation $v: K \rightarrow[0, \infty]$ with $v(d)=2$. We show first that for every $n \in \mathbb{N}$ there exists some $c \in K^{\times}$such that

$$
d^{-1} \leqslant(1+c p)^{n} \leqslant d
$$

holds for all $p \in P$. From (P4) we get by induction $P+\cdots+P \subset d^{n} P$, if the sum $P+\cdots+P$ contains at most $2^{n}$ summands. For $c \in P$, we therefore have $(1+c p)^{n} \in 1+c P+\cdots+c P \subset 1+c d^{n} P$. Replacing $c$ by $c d^{-n}$, the inequality (35) turns into $d^{-1} \leqslant 1+c p \leqslant d$ for some $c \in K^{\times}$and $p \in P$. By Lemma 3.1 (with $a=1$ ), this reduces to the single inequality $1+c p \leqslant d$. This is equivalent to $(1+c p) d^{-1} \in \widetilde{P}$, i. e. $(1+c p) d^{-1} P \subset P$. By $(\mathrm{P} 4)$, the latter is satisfied for $c=1$. 
Using $v$, we define $f: K \rightarrow \mathbb{R}$ by

$$
f(a):=\left\{\begin{array}{ll}
1 & \text { for } a \in P \\
2-v(a) & \text { for } a \in d P \backslash P \\
0 & \text { for } a \notin d P
\end{array} \backslash\right.
$$

and prove the uniform continuity of $f$. Let $\varepsilon>0$ be given. We have to find $c \in K^{\times}$so that $\left|f(a)-f\left(a^{\prime}\right)\right|<\varepsilon$ holds for all $a, a^{\prime} \in K$ with $a-a^{\prime} \in c P$. By symmetry, we can assume that $a \in d P \backslash P$.

Case I: $a^{\prime} \in P$. For $c \in d^{-1} P$, this implies that $a^{\prime} \notin d^{-1} P$, since otherwise, $a=\left(a-a^{\prime}\right)+a^{\prime} \in$ $d^{-1} P+d^{-1} P \subset P$. Hence if $n \in \mathbb{N}$, the inequality (35) yields an element $c \in K^{\times}$with $\left(1+\frac{c}{a^{\prime}} p\right)^{n} \leqslant d$ for all $p \in P$. For $a-a^{\prime}=c p$, we thus have $a<\frac{a}{a^{\prime}}$, hence $d^{-1}<1 \leqslant a^{n} \leqslant\left(\frac{a}{a^{\prime}}\right)^{n}=\left(1+\frac{c}{a^{\prime}} p\right)^{n} \leqslant d$. So we get $2^{-1} \leqslant v\left(a^{n}\right) \leqslant 2$, and thus $2^{-\frac{1}{n}} \leqslant v(a) \leqslant 2^{\frac{1}{n}}$. Consequently, $\left|f(a)-f\left(a^{\prime}\right)\right|=|(2-v(a))-1|=$ $|v(a)-1|<\varepsilon$ holds for sufficiently large $n$.

Case II: $a^{\prime} \notin d P$. For any $n \in \mathbb{N}$, the inequality (35) shows that there exists some $c \in K^{\times}$with $d^{-1} \leqslant\left(1+\frac{c}{a^{\prime}} p\right)^{n}$ for all $p \in P$. For $a-a^{\prime}=c p$, this gives $d^{-1} \leqslant\left(1+\frac{c}{a^{\prime}} p\right)^{n}=\left(\frac{a}{a^{\prime}}\right)^{n} \leqslant\left(\frac{a}{d}\right)^{n} \leqslant 1<d$. Therefore, $d^{n-1} \leqslant a^{n} \leqslant d^{n+1}$, hence $2^{n-1} \leqslant v(a)^{n} \leqslant 2^{n+1}$, and thus $2^{\frac{n-1}{n}} \leqslant v(a) \leqslant 2^{\frac{n+1}{n}}$. For sufficiently large $n$, we get $\left|f(a)-f\left(a^{\prime}\right)\right|=|v(a)-2|<\varepsilon$.

Case III: $a^{\prime} \in d P \backslash P$. Again by (35), for any $n \in \mathbb{N}$, there exists $c \in K^{\times}$with $d^{-1} \leqslant$ $\left(1+\frac{c}{a^{\prime}} p\right)^{n} \leqslant d$ for all $p \in P$. For $a-a^{\prime}=c p$, we get $d^{-1} \leqslant\left(\frac{a}{a^{\prime}}\right)^{n} \leqslant d$ for all $p \in P$. This gives $2^{-1} \leqslant v(a)^{n} v\left(a^{\prime}\right)^{-n} \leqslant 2$, hence $2^{-\frac{1}{n}} \leqslant \frac{v(a)}{v\left(a^{\prime}\right)} \leqslant 2^{\frac{1}{n}}$. For sufficiently large $n$, it follows that $\left|f(a)-f\left(a^{\prime}\right)\right|=\left|v(a)-v\left(a^{\prime}\right)\right|<\varepsilon$.

Note that $f \in \mathcal{C}_{P}(K)$ implies that $|f| \in \mathcal{C}_{P}(K)$. Therefore, every $f \in \mathcal{C}_{P}(K)$ admits a decomposition

$$
f=f_{+}-f_{-}
$$

with $f_{+}:=\frac{1}{2}(|f|+f)$ and $f_{-}:=\frac{1}{2}(|f|-f)$, so that $|f|=f_{+}+f_{-}$and $f_{+}, f_{-} \geqslant 0$. For $f \in \mathcal{C}_{P}(K)$ and $a \in K$, we define $f^{a} \in \mathcal{C}_{P}(K)$ by

$$
f^{a}(b):=f(b-a) .
$$

Definition 4.2. Let $K$ be a field with a compact prime $P$. A linear form $I: \mathcal{C}_{P}(K) \rightarrow \mathbb{R}$ is said to be a Haar integral if $I(f)>0$ holds for $f>0$, and $I\left(f^{a}\right)=I(f)$ for all $a \in K$.

So the Haar integral is linear (like any integral) and monotonous $(f<g \Rightarrow I(f)<I(g))$, and invariant under translations. We will show first that a Haar integral exists and is unique up to a constant. Our proof is similar to [14], but does not assume that the topology of $K$ given by the compact prime $P$ is locally compact. In section 6 , we will show that the constant can be chosen in a canonical way.

For $f, g \in \mathcal{C}_{P}^{+}(K)$, let $f: g$ be the infimum of all sums $r_{1}+\cdots+r_{n}$ with $r_{1}, \ldots, r_{n}>0$ in $\mathbb{R}$, such that $f \leqslant r_{1} g^{a_{1}}+\cdots+r_{n} g^{a_{n}}$ holds for suitable $a_{1}, \ldots, a_{n} \in K$.

Proposition 4.3. Let $K$ be a field with a compact prime $P$. Every function $f \in \mathcal{C}_{P}(K)$ is bounded. For $f, g \in \mathcal{C}_{P}^{+}(K)$, we have $0<f: g<\infty$.

Proof. First let $f \in \mathcal{C}_{P}(K)$ and $b \in K^{\times}$be given such that $f(a)=0$ holds for $a \notin b P$. For every $\varepsilon>0$, there exists some $c \in K^{\times}$such that $\left|f(a)-f\left(a^{\prime}\right)\right|<\varepsilon$ holds for $a-a^{\prime} \in c P$. Since $P$ is compact, there are $a_{1}, \ldots, a_{n} \in K$ with $b P \subset\left(a_{1}+c P\right) \cup \cdots \cup\left(a_{n}+c P\right)$. Hence $f$ is bounded. 
Next let $f, g \in \mathcal{C}_{P}^{+}(K)$ be given. Choose $a_{0} \in K$ with $g\left(a_{0}\right)>0$. Then we find $\varepsilon>0$ in $\mathbb{R}$ and $c \in K^{\times}$with $g(a) \geqslant \varepsilon$ for $a-a_{0} \in c P$. Assume that $f(a)=0$ for $a \notin b P$ and $a_{1}, \ldots, a_{n} \in K$ with $b P \subset\left(a_{1}+c P\right) \cup \cdots \cup\left(a_{n}+c P\right)$. Then there exists some $m \in \mathbb{N}$ with $f(a) \leqslant m \varepsilon$ for all $a \in b P$. Hence $f \leqslant m g^{a_{1}-a_{0}}+\cdots+m g^{a_{n}-a_{0}}$, which gives $f: g<\infty$. Furthermore, $g(a) \leqslant r$ for all $a \in K$ and a real $r>0$. Now if $f(a)>0$, we obtain $f: g \geqslant f(a) r^{-1}>0$.

Lemma 4.1. Let $K$ be a field with a compact prime $P$. Let $b \in K^{\times}$and $f, g \in \mathcal{C}_{P}^{+}(K)$ satisfy $f(a)=0$ for $a \notin b P$ and $g(a) \geqslant \varepsilon$ for some $\varepsilon>0$ in $\mathbb{R}$ and $a \in b(P+P)$. Then $h:=\frac{f}{g} \in \mathcal{C}_{P}^{+}(K)$, where $h(a):=0$ for $a \notin b P$.

Proof. For all $a, a^{\prime} \in K$, we have:

$$
\begin{aligned}
\left|h(a)-h\left(a^{\prime}\right)\right| & =\frac{\left|f(a) g\left(a^{\prime}\right)-g(a) f\left(a^{\prime}\right)\right|}{g(a) g\left(a^{\prime}\right)} \\
& =\frac{\left|\left(f(a)-f\left(a^{\prime}\right)\right) g\left(a^{\prime}\right)+f\left(a^{\prime}\right)\left(g\left(a^{\prime}\right)-g(a)\right)\right|}{g(a) g\left(a^{\prime}\right)} \\
& \leqslant \frac{\left|f(a)-f\left(a^{\prime}\right)\right|}{g(a)}+\frac{f\left(a^{\prime}\right)}{g\left(a^{\prime}\right)} \cdot \frac{\left|g(a)-g\left(a^{\prime}\right)\right|}{g(a)} .
\end{aligned}
$$

For $a, a^{\prime} \notin b P$, this expression vanishes. Otherwise, if $a-a^{\prime} \in b P$, then $a, a^{\prime} \in b P+b P$. Hence

$$
\left|h(a)-h\left(a^{\prime}\right)\right| \leqslant \varepsilon^{-1}\left|f(a)-f\left(a^{\prime}\right)\right|+\varepsilon^{-2} f\left(a^{\prime}\right) \cdot\left|g(a)-g\left(a^{\prime}\right)\right|
$$

for all $a, a^{\prime} \in K$. Since $f$ is bounded by Proposition 4.3, this gives $h \in \mathcal{C}_{P}^{+}(K)$.

Proposition 4.4. Let $K$ be a field with a compact prime $P$. For every $f \in \mathcal{C}_{P}^{+}(K)$ and $c \in K^{\times}$, there are $f_{1}, \ldots, f_{n} \in \mathcal{C}_{P}^{+}(K)$ and $a_{1}, \ldots, a_{n} \in K$ such that $f_{i}(a)=0$ holds for $a-a_{i} \notin c P$, so that $f=f_{1}+\cdots+f_{n}$.

Proof. Assume that $f(a)=0$ holds for $a \notin b P$, where $b \in K^{\times}$. By Proposition 4.2, there exists some $f^{\prime} \in \mathcal{C}_{P}^{+}(K)$ which satisfies $f^{\prime}(a)=1$ for $a \in b(P+P)$. Furthermore, there exists some $g \in \mathcal{C}_{P}^{+}(K)$ that vanishes outside $c P$. By Proposition 4.3, we have $\left(f+f^{\prime}\right): g<\infty$. Hence there are real numbers $r_{1}, \ldots, r_{n}>0$ and $a_{1}, \ldots, a_{n} \in K$ with $f+f^{\prime} \leqslant r_{1} g^{a_{1}}+\cdots+r_{n} g^{a_{n}}$. By Lemma 4.1, $h:=\frac{f}{r_{1} g^{a_{1}}+\cdots+r_{n} g^{a_{n}}} \in \mathcal{C}_{P}^{+}(K)$, whence $f=r_{1} h g^{a_{1}}+\cdots+r_{n} h g^{a_{n}}$.

\section{$5 \quad$ Existence and Uniqueness}

Now we are ready to prove existence and uniqueness of the Haar integral.

Proposition 5.1. For every compact prime $P$ of a field $K$, there exists a Haar integral.

Proof. By (36), it is enough to find a map $I: \mathcal{C}_{P}^{+}(K) \rightarrow(0, \infty)$ which satisfies $I(r f+s g)=$ $r I(f)+s I(g)$ for $r, s \geqslant 0$ and $I\left(f^{a}\right)=I(f)$ for $a \in K$. For $r>0$ in $\mathbb{R}$ and $f, g \in \mathcal{C}_{P}^{+}(K)$, we have $(r f: g)=r(f: g)$. Furthermore, for $f, g, h \in \mathcal{C}_{P}^{+}(K)$,

$$
(f: g)(g: h) \geqslant f: h
$$


In fact, assume that $f \leqslant r_{1} g^{a_{1}}+\cdots+r_{n} g^{a_{n}}$ and $g \leqslant s_{1} h^{b_{1}}+\cdots+s_{m} h^{b_{m}}$. Then $f \leqslant \sum_{i=1}^{n} r_{i}\left(\sum_{j=1}^{m} s_{j} h^{b_{j}}\right)^{a_{i}}=\sum_{i=1}^{n} \sum_{j=1}^{m} r_{i} s_{j} h^{b_{j}+a_{i}}$, and thus $f: h \leqslant\left(\sum_{i=1}^{n} a_{i}\right)\left(\sum_{j=1}^{m} b_{j}\right)$.

To determine $I(f)$, let $f_{0} \in \mathcal{C}_{P}^{+}(K)$ be fixed. Then $f: f_{0}$ can be regarded as a first approximation of $I(f): I\left(f_{0}\right)$. An improvement is given by the function $I_{g}: \mathcal{C}_{P}^{+}(K) \rightarrow(0, \infty)$ with

$$
I_{g}(f):=\frac{f: g}{f_{0}: g}
$$

for small $g \in \mathcal{C}_{P}^{+}(K)$. By (38), we have

$$
\left(f_{0}: f\right)^{-1} \leqslant I_{g}(f) \leqslant f: f_{0} .
$$

For $b \in K^{\times}$, let $V_{b}$ be the set of all $I_{g}$ with $g(a)=0$ for $a \notin b P$. By Proposition $4.2, V_{b} \neq \varnothing$ for all $b \in K^{\times}$. By (40), we can regard $I_{g}$ as a point of the compact space

$$
X:=\prod_{f \in \mathcal{C}_{P}^{+}(K)}\left[\left(f_{0}: f\right)^{-1}, f: f_{0}\right] .
$$

As the closed sets $\overline{V_{b}} \subset X$ form a chain, their intersection is non-empty. Hence there exists some $I \in \bigcap_{b \in K^{\times}} \overline{V_{b}}$. Consequently, for every $b \in K^{\times}$and arbitrary $f_{1}, \ldots, f_{n} \in \mathcal{C}_{P}^{+}(K)$ and $\varepsilon>0$ in $\mathbb{R}$, there exists some $g \in \mathcal{C}_{P}^{+}(K)$ with $g(a)=0$ for $a \notin b P$, so that for $i \in\{1, \ldots, n\}$,

$$
\left|I\left(f_{i}\right)-I_{g}\left(f_{i}\right)\right|<\varepsilon .
$$

We will prove that $I$ is a Haar integral. Firstly,

$$
\left(f_{1}+f_{2}\right): g \leqslant\left(f_{1}: g\right)+\left(f_{2}: g\right)
$$

implies that $I_{g}\left(f_{1}+f_{2}\right) \leqslant I_{g}\left(f_{1}\right)+I_{g}\left(f_{2}\right)$ holds for $f_{1}, f_{2}, g \in \mathcal{C}_{P}^{+}(K)$. Hence (42) gives

$$
I\left(f_{1}+f_{2}\right) \leqslant I\left(f_{1}\right)+I\left(f_{2}\right)
$$

for all $f_{1}, f_{2} \in \mathcal{C}_{P}^{+}(K)$. For $0<r<\infty$ and $f, g \in \mathcal{C}_{P}^{+}(K)$, we have $(r f: g)=r(f: g)$, i. e. $I_{g}(r f)=r I_{g}(f)$, and thus $I(r f)=r I(f)$, again by (42). Similarly, $I_{g}\left(f^{a}\right)=I_{g}(f)$ for all $a \in K$, hence $I\left(f^{a}\right)=I(f)$. From (41) and (42), it follows that $I$ is monotonous. Thus it remains to show that the inequality (43) is an equation.

Let $f_{1}, f_{2}, g \in \mathcal{C}_{P}^{+}(K)$ be given. Then there exists some $b \in K^{\times}$such that $f_{1}+f_{2}$ vanishes outside $b P$. By Proposition 4.2, we find $f_{3} \in \mathcal{C}_{P}^{+}(K)$ with $f_{3}(a)=1$ for $a \in b(P+P)$. For an arbitrary $\varepsilon>0$, define $f:=f_{1}+f_{2}+\varepsilon f_{3}$ and $h_{j}:=\frac{f_{j}}{f}$ for $j \in\{1,2\}$. By Lemma 4.1, this gives $h_{j} \in \mathcal{C}_{P}^{+}(K)$. Hence there exists some $c \in K^{\times}$such that $\left|h_{j}(a)-h_{j}\left(a^{\prime}\right)\right|<\varepsilon$ holds for $a-a^{\prime} \in c P$ and $j \in\{1,2\}$. Choose $g \in \mathcal{C}_{P}^{+}(K)$ with $g(a)=0$ for $a \notin c P$, and

$$
f \leqslant r_{1} g^{a_{1}}+\cdots+r_{n} g^{a_{n}} .
$$

Then $f_{j}(a)=f(a) h_{j}(a) \leqslant \sum_{i=1}^{n} r_{i} g^{a_{i}}(a)\left(h_{j}\left(a_{i}\right)+\varepsilon\right)$, hence

$$
f_{j}: g \leqslant \sum_{i=1}^{n} r_{i}\left(h_{j}\left(a_{i}\right)+\varepsilon\right) .
$$

This implies $\left(f_{1}: g\right)+\left(f_{2}: g\right) \leqslant \sum_{i=1}^{n} r_{i}(1+2 \varepsilon)$ since $h_{1}+h_{2} \leqslant 1$. So we get $\left(f_{1}: g\right)+\left(f_{2}: g\right) \leqslant(f:$ $g)(1+2 \varepsilon)$, and thus $I_{g}\left(f_{1}\right)+I_{g}\left(f_{2}\right) \leqslant I_{g}(f) \cdot(1+2 \varepsilon)$. By $(42)$, this gives $I\left(f_{1}\right)+I\left(f_{2}\right) \leqslant I(f)(1+2 \varepsilon)$. Using (43), we get $I\left(f_{1}\right)+I\left(f_{2}\right) \leqslant\left(I\left(f_{1}+f_{2}\right)+\varepsilon I\left(f_{3}\right)\right)(1+2 \varepsilon)$ for all $\varepsilon$, hence $I\left(f_{1}\right)+I\left(f_{2}\right) \leqslant I\left(f_{1}+f_{2}\right)$. 
Proposition 5.2. Let $K$ be a field with a compact prime $P$. For every $f_{0} \in \mathcal{C}_{P}^{+}(K)$, there exists a unique Haar integral $I: \mathcal{C}_{P}(K) \rightarrow \mathbb{R}$ with $I\left(f_{0}\right)=1$.

Proof. Existence follows by Proposition 5.1. Therefore, let $I$ be a Haar integral, and $f_{0} \in \mathcal{C}_{P}^{+}(K)$. Then $r I$ is a Haar integral for any $r>0$ in $\mathbb{R}$. Assume first that $f, g \in \mathcal{C}_{P}^{+}(K)$ and $f \leqslant \sum_{i=1}^{n} r_{i} g^{a_{i}}$ with $r_{i}>0$ and $a_{i} \in K$. Then $I(f) \leqslant \sum_{i=1}^{n} r_{i} I(g)$, hence

$$
\frac{I(f)}{I(g)} \leqslant f: g \text {. }
$$

For $f \in \mathcal{C}_{P}^{+}(K)$ and $\varepsilon>0$, there exists some $b \in K^{\times}$which satisfies $\left|f(a)-f\left(a^{\prime}\right)\right|<\varepsilon$ for $a-a^{\prime} \in b P$. Choose $g \in \mathcal{C}_{P}^{+}(K)$ with $g(a)=0$ for $a \notin b P$. Passing from $g(a)$ to $g(a)+g(-a)$, we can assume that $g(a)=g(-a)$ for all $a \in K$. Therefore, if $a, a^{\prime} \in K$, we have $f\left(a^{\prime}\right) g^{a}\left(a^{\prime}\right) \geqslant(f(a)-\varepsilon) g^{a}\left(a^{\prime}\right)$, hence $I\left(f g^{a}\right) \geqslant(f(a)-\varepsilon) I(g)$, and thus

$$
f(a) \leqslant \varepsilon+\frac{I\left(f g^{a}\right)}{I(g)} .
$$

For every $\delta>0$, there is some $c \in K^{\times}$with $\left|g(a)-g\left(a^{\prime}\right)\right|<\delta$ for $a-a^{\prime} \in c P$. By Proposition 4.4, there are elements $f_{1}, \ldots, f_{n} \in \mathcal{C}_{P}^{+}(K)$ and $a_{1}, \ldots, a_{n} \in K$ with $f=f_{1}+\cdots+f_{n}$ and $f_{i}\left(a^{\prime}\right)=0$ for $a^{\prime}-a_{i} \notin c P$. This gives $f_{i}\left(a^{\prime}\right) g\left(a^{\prime}-a\right) \leqslant f_{i}\left(a^{\prime}\right)\left(g\left(a_{i}-a\right)+\delta\right)$ for all $a, a^{\prime} \in K$, hence

$$
I\left(f g^{a}\right)=\sum_{i=1}^{n} I\left(f_{i} g^{a}\right) \leqslant \sum_{i=1}^{n} I\left(f_{i}\right)\left(g^{a}\left(a_{i}\right)+\delta\right)=\sum_{i=1}^{n} I\left(f_{i}\right)\left(g^{a_{i}}(a)+\delta\right) .
$$

By Proposition 4.2, there exists some $h \in \mathcal{C}_{P}^{+}(K)$ such that $h\left(a^{\prime}\right)=1$ holds for all $a^{\prime}$ with $f\left(a^{\prime}\right)+$ $f_{0}\left(a^{\prime}\right)>0$. Then $(45)$ and (46) yield

$$
f \leqslant \varepsilon h+\sum_{i=1}^{n} \frac{I\left(f_{i}\right)}{I(g)}\left(g^{a_{i}}+\delta h\right)=\left(\varepsilon+\frac{I(f)}{I(g)} \delta\right) h+\sum_{i=1}^{n} \frac{I\left(f_{i}\right)}{I(g)} g^{a_{i}} .
$$

So we get $f: g \leqslant \varepsilon(h: g)+\frac{I(f)}{I(g)}$, and by virtue of $(38)$ and $(44)$,

$$
\frac{f: g}{f_{0}: g} \leqslant \varepsilon \cdot \frac{h: g}{f_{0}: g}+\frac{I(f)}{I(g)\left(f_{0}: g\right)} \leqslant \varepsilon\left(h: f_{0}\right)+\frac{I(f)}{I\left(f_{0}\right)} .
$$

Similarly,

$$
\frac{f_{0}: g}{f: g} \leqslant \varepsilon(h: f)+\frac{I\left(f_{0}\right)}{I(f)}=\frac{I\left(f_{0}\right)}{I(f)}\left(1+\varepsilon(h: f) \frac{I(f)}{I\left(f_{0}\right)}\right),
$$

and thus

$$
\frac{I(f)}{I\left(f_{0}\right)}\left(1+\varepsilon(h: f) \frac{I(f)}{I\left(f_{0}\right)}\right)^{-1} \leqslant \frac{f: g}{f_{0}: g} \leqslant \varepsilon\left(h: f_{0}\right)+\frac{I(f)}{I\left(f_{0}\right)} .
$$

This implies that the quotient $\frac{f: g}{f_{0}: g}$ converges to $\frac{I(f)}{I\left(f_{0}\right)}$ for $b \rightarrow 0$, which shows that $I(f)$ is unique for $I\left(f_{0}\right)=1$.

\section{The normalized Haar integral}

Let $K$ be a field with a compact prime $P$. By Proposition 5.2, there exists a Haar integral $I: \mathcal{C}_{P}(K) \rightarrow$ $\mathbb{R}$, unique up to a real constant. Furthermore, the proof of Proposition 5.2 yields an explicit determination of the Haar integrals. In fact, for any $b \in K^{\times}$, let $L(b)$ be the set of $g \in \mathcal{C}_{P}^{+}(K)$ which vanish 
outside $b P$. Moreover, we set

$$
L_{s}(b):=\{g \in L(b) \mid \forall a \in K: g(-a)=g(a)\} .
$$

For $f, f_{0} \in \mathcal{C}_{P}^{+}(K)$ and every $\varepsilon>0$ in $\mathbb{R}$, There exists some $b \in K^{\times}$such that

$$
\left|\frac{I(f)}{I\left(f_{0}\right)}-\frac{f: g}{f_{0}: g}\right|<\varepsilon
$$

for all $g \in L_{s}(b)$. In this way, the quotient $\frac{I(f)}{I\left(f_{0}\right)}$ can be approximated by the expressions (39) with $g \in L_{s}(b)$.

For any $c \in K^{\times}$, consider the $\mathbb{R}$-linear map

$$
\rho_{c}: \mathcal{C}_{P}(K) \rightarrow \mathcal{C}_{P}(K)
$$

with $\rho_{c}(f)(a):=f\left(c^{-1} a\right)$ for all $f \in \mathcal{C}_{P}(K)$ and $a \in K$. Since $f(K \backslash b P)=0 \Leftrightarrow \rho_{c}(f)(K \backslash c b P)=0$, we have

$$
\rho_{c}(L(b))=L(c b) .
$$

Similarly, the uniform continuity is preserved under the map $\rho_{c}$. Furthermore, $\rho_{c}$ is monotonous, i. e. for $f, g \in \mathcal{C}_{P}(K)$,

$$
f \leqslant g \Longleftrightarrow \rho_{c}(f) \leqslant \rho_{c}(g) .
$$

Since $\rho_{c}$ is invertible, it induces a bijection

$$
\rho_{c}: \mathcal{C}_{P}^{+}(K) \stackrel{\sim}{\longrightarrow} \mathcal{C}_{P}^{+}(K) .
$$

For $a, b \in K$ and $f \in \mathcal{C}_{P}(K)$, we finally have $\rho_{c}\left(f^{a}\right)(b)=f^{a}\left(c^{-1} b\right)=f\left(c^{-1} b-a\right)=f\left(c^{-1}(b-c a)\right)=$ $\rho_{c}(f)(b-c a)=\rho_{c}(f)^{c a}(b)$, hence

$$
\rho_{c}\left(f^{a}\right)=\rho_{c}(f)^{c a} .
$$

Consequently, for any Haar integral $I: \mathcal{C}_{P}(K) \rightarrow \mathcal{C}_{P}(K)$ and $c \in K^{\times}$, it follows that $I \rho_{c}$ is again a Haar integral. So there is a real function $v_{P}: K \rightarrow[0, \infty]$ with $v_{P}(0):=0$ and

$$
I \rho_{c}=v_{P}(c) I
$$

for $c \neq 0$, which does not depend on the choice of the Haar integrals $I$.

Now we prove that $v_{P}$ is a pseudo-valuation. Using $v_{P}$, the Haar integral can be normalized in a natural way.

Proposition 6.1. Let $K$ be a field with a compact prime $P$. Then the uniquely defined function $v_{P}: K \rightarrow[0, \infty]$ in $(54)$ is a pseudo-valuation with respect to $P$.

Proof. From (54) we infer that $v_{P}$ is multiplicative: $v_{P}(b c)=v_{P}(b) v_{P}(c)$ for all $b, c \in K$. In fact, for $b, c \in K^{\times}$and a Haar integral $I, v_{P}(b c) I=I \rho_{b c}=I \rho_{b} \rho_{c}=v_{P}(b) I \rho_{c}=v_{P}(b) v_{P}(c) I$. Similarly, $v_{P}(1)=1$.

To prove that $v_{P}$ is monotonous, we use $I$ to define a function $v_{I}: K \rightarrow[0, \infty)$ which is related to $v_{P}$. (In contrast to $v_{P}$, however, $v_{I}$ depends on $I$.) For $b \in K$, we set $U(b):=\left\{f \in \mathcal{C}_{P}^{+}(K) \mid \forall a \in\right.$ $b \widetilde{P}: f(a)=1\}$, and

$$
v_{I}(b):=\inf _{f \in U(b)} I(f)
$$


Although the function (55) is not multiplicative, in general, it is always monotonous. In fact, if $b, c \in K$ with $b \leqslant c$, then $U(b) \supset U(c)$, and therefore, $v_{I}(b) \leqslant v_{I}(c)$.

Before we compare $v_{P}$ with $v_{I}$, we prove, in analogy to (50), that

$$
\rho_{c}(U(b))=U(c b)
$$

holds for $b, c \in K^{\times}$. This follows by the equivalence $\rho_{c}(f) \in U(c b) \Leftrightarrow f \in U(b)$ for all $f \in \mathcal{C}_{P}^{+}(K)$. For $b, c \in K^{\times}$, we have the equation $v_{P}(c) \cdot \inf _{f \in U(b)} I(f)=\inf _{f \in U(b)} I\left(\rho_{c}(f)\right)=\inf _{f \in U(c b)} I(f)$, which gives

$$
v_{I}(c b)=v_{P}(c) \cdot v_{I}(b) .
$$

In particular, this gives $v_{I}(c)=v_{P}(c) \cdot v_{I}(1)$, i. e.

$$
v_{I}=v_{I}(1) \cdot v_{P}
$$

By Proposition 4.2, we find a function $g \in L(1)$ with $g \leqslant 1$. For every $f \in U(1)$, we get $f \geqslant g$, hence $I(f) \geqslant I(g)$, and thus $v_{I}(1) \geqslant I(g)>0$. By (58), the monotonous property of $v_{I}$ carries over to $v_{P}$, i. e. $v_{P}$ is a pseudo-valuation.

The fact that $v_{I}$ depends on $I$ enables us to normalize the Haar Integral:

Theorem 6.1. Let $K$ be a field with a compact prime $P$. There exists a unique Haar integral $J$ with $v_{J}=v_{P}$.

Proof. Let $I$ be a Haar integral, and $J=r I$ with $r>0$ in $\mathbb{R}$. Then $v_{r I}(1)=r v_{I}(1)$. By Eq. (58), the condition $v_{J}=v_{P}$ is equivalent to $v_{J}(1)=1$, that is, $r=v_{I}(1)^{-1}$.

In the sequel, we simply call $J$ the integral and $v_{P}=v_{J}$ the normed pseudo-valuation with respect to $P$. Then $(55)$ shows that $v_{P}(b)$ measures the content of $b \widetilde{P}$. Our normalization thus fixes the content of $\widetilde{P}$ to 1 .

Next we determine the pseudo-valuations of a compact prime. First, let $P$ be an arbitrary prime. We call a pseudo-valuation $v: K \rightarrow[0, \infty]$ with respect to $P$ trivial if the values of $v$ belong to $\{0,1, \infty\}$.

Proposition 6.2. Let $K$ be a field with a prime $P$. There is a natural bijection between the primes $Q \subset P$ and the trivial pseudo-valuations $v: K \rightarrow[0, \infty]$ with respect to $P$.

Proof. First, let $v: K \rightarrow[0, \infty]$ be a trivial pseudo-valuation with respect to $P$. By Proposition 2.1, the primes $Q \subset P$ correspond to the multiplicatively closed subsets $U \subset K$ with $\widetilde{P} \subset U$, where $U=\widetilde{Q}$. For such a $Q$, we define $v$ by

$$
v(a):= \begin{cases}0 & \text { for } a \in Q \\ 1 & \text { for } a \in \partial Q \\ \infty & \text { for } a \in K \backslash \widetilde{Q} .\end{cases}
$$

Then $v$ is monotonous with $v(0)=0$ and $v(1)=1$. For $a, b \in K$ with $v(a)=1$, we have $b \in$ $Q \Leftrightarrow a b \in Q$ and $b \in \widetilde{Q} \Leftrightarrow a b \in \widetilde{Q}$. Hence $v$ is a pseudo-valuation by Definition 3.1. Conversely, let $v: K \rightarrow[0, \infty]$ be a trivial pseudo-valuation with respect to $P$. We set $U:=\{a \in K \mid v(a)<\infty\}$. Then $U$ is multiplicatively closed with $\widetilde{P} \subset U$. The corresponding prime $Q \subset P$ satisfies $a \in \widetilde{Q} \Leftrightarrow v(a) \leqslant 1$, and by (31), $a \in Q \Leftrightarrow a^{-1} \notin \widetilde{Q} \Leftrightarrow v\left(a^{-1}\right)=\infty \Leftrightarrow v(a)=0$ for $a \in K^{\times}$, in accordance with (59). 
Proposition 6.3. Let $K$ be a field with a finite, non-trivial pseudo-valuation $v: K \rightarrow[0, \infty]$ with respect to a prime $P$. Then $Q:=\{a \in K \mid v(a)<1\} \subset P$ is a minimal prime.

Proof. For $a, b \in Q \cap K^{\times}$, we have $v(a b)=v(a) v(b)<1$, hence $a b \in Q$. If $a, b \notin Q$, then $v(a), v(b) \geqslant 1$, which gives $v(a b)=v(a) v(b) \geqslant 1$, i. e. $a b \notin Q$. This proves (P1), (P2), and (P3). Thus $Q \subset P$ implies that $Q$ is a prime. Now assume that $a, b \in Q \cap K^{\times}$. Then there exists some $n \in \mathbb{N}$ with $v\left(a^{n}\right)=v(a)^{n}<v(b)$. Hence $a^{n}<b$, and thus $a^{n} \in b P$. So $Q$ is minimal by Proposition 2.2 .

Corollary. Every compact prime $P \neq 0$ of a field $K$ contains a minimal prime.

Proof. Proposition 6.1 implies that $v_{P}$ is a finite pseudo-valuation, and $v_{P}$ is non-trivial since $P \neq 0$. Now Proposition 6.3 completes the proof.

\section{$7 \quad$ Krull primes}

By Proposition 3.4, every minimal prime $P$ of a field $K$ gives rise to a family of $P$-valuations. Conversely,

Proposition 7.1. Let $K$ be a field with a prime $P \neq 0$ and a $P$-valuation $v: K \rightarrow[0, \infty]$. Then $P$ is minimal.

Proof. Let $a, b \in P \cap K^{\times}$be given. Then $v\left(a^{-1}\right)<\infty$ implies that $v(a) \neq 0$, and $a<1$ yields $0<v(a)<1$. So there is an $n \in \mathbb{N}$ with $v\left(a^{n}\right)=v(a)^{n} \leqslant v(b)$. Hence $a^{n} \leqslant b$. By Proposition 2.2, we infer that $P$ is minimal.

In algebraic number theory, the distinction between finite and infinite primes is crucial. For an arbitrary field, we have to distinguish between algebraic and transcendental primes. First, we prove

Lemma 7.1. A prime $P$ of a field $K$ is algebraic if and only if $\widetilde{P}$ is a subring of $K$.

Proof. If $P$ is algebraic and $a, b \in \widetilde{P}$, then $(a+b) P \subset P+P \subset P$, hence $a+b \in \widetilde{P}$. Therefore, $\widetilde{P}$ is a subring. Conversely, assume that $\widetilde{P}+\widetilde{P} \subset \widetilde{P}$ and $a, b \in P$, say, $a \leqslant b$. Then $a b^{-1} \in \widetilde{P}$, which implies that $a b^{-1}+1 \in \widetilde{P}$. Hence $a+b=\left(a b^{-1}+1\right) b \in P$. So we get $P+P \subset P$, and thus $P$ is algebraic.

Proposition 7.2. For every prime $P$ of a field $K$, there is a largest algebraic prime $P_{\circ} \subset P$.

Proof. The subring of $K$ generated by $\widetilde{P}$ is

$$
U:=\left\{a_{1}+\cdots+a_{n} \mid a_{i} \in \widetilde{P}\right\} .
$$

By Proposition 2.1, $U$ corresponds to a prime $P_{\circ} \subset P$ with $\widetilde{P_{\circ}}=U$. By Lemma 7.1, this prime meets the requirement.

There is a close relationship between $P_{\circ}$ and the open subset $P^{\circ}$ of $P$ given by Proposition 3.1. First, we prove

Proposition 7.3. If $P$ is a prime of a field $K$, then $P^{\circ}$ is a prime, and $P^{\circ \circ}=P^{\circ}$. 
Proof. Clearly, $P^{\circ}$ satisfies (P1) and (P2). Let $a, b \notin P^{\circ}$ be given. For any $n \in \mathbb{N}$, there are elements $p_{n}, q_{n}, r_{n}, s_{n} \in P$ with $a^{n}\left(p_{n}+q_{n}\right) \notin P$ and $b^{n}\left(r_{n}+s_{n}\right) \notin P$. Hence $a^{n} b^{n}\left(p_{n}+q_{n}\right)\left(r_{n}+s_{n}\right) \notin P$, and thus $a^{n} b^{n}(P+P+P+P) \not \subset P$ for all $n \in \mathbb{N}$. If $a b \in P^{\circ}$, there exists some $n \in \mathbb{N}$ with $(a b)^{n}(P+P) \subset P$, whence $(a b)^{2 n}(P+P+P+P) \subset(a b)^{n}(P+P) \subset P$, a contradiction. This proves (P3) for $P^{\circ}$. To verify (P4), choose $d \in K^{\times}$with $P+P \subset d P$. We show that $P^{\circ}+P^{\circ} \subset d P^{\circ}$. To this end, assume that $a, b \in P^{\circ}$, say, $a^{n}(P+P) \subset P$ and $b^{n}(P+P) \subset P$. Then $(a+b)^{2 n}(P+P)=$ $\sum_{i=0}^{2 n}\left(\begin{array}{c}2 n \\ i\end{array}\right) a^{i} b^{2 n-i}(P+P) \subset \sum_{i=0}^{2 n}\left(\begin{array}{c}2 n \\ i\end{array}\right) P \subset \sum_{i=1}^{2^{2 n}} P \subset d^{2 n} P$, which gives $(a+b) d^{-1} \in P^{\circ}$. Whence $P^{\circ}+P^{\circ} \subset d P^{\circ}$.

Finally, assume that $a \in P^{\circ}$, say, $a^{n}(P+P) \subset P$. Then $P+P \subset a^{-n} P$, and by the above, this implies that $P^{\circ}+P^{\circ} \subset a^{-n} P^{\circ}$. Hence $a^{n}\left(P^{\circ}+P^{\circ}\right) \subset P^{\circ}$, and thus $a \in P^{\circ \circ}$.

Primes $P$ with $P=P^{\circ}$ were first considered by Krull [7]. We call them Krull primes. By Proposition 3.1, every minimal prime is a Krull prime. The connection to algebraic primes is given by

Proposition 7.4. Let $K$ be a field with a prime $P$. Then every prime $Q \subsetneq P^{\circ}$ of $K$ is algebraic.

Proof. Choose $a \in P^{\circ} \backslash Q$. So there is an $n \in \mathbb{N}$ with $a^{n}(P+P) \subset P$. Let $b \in Q$ be arbitrary. Then $a \notin Q$ implies that $a^{-1} \in \widetilde{Q}$, hence $a^{-n-1} b \in Q$. This gives $b(\widetilde{P}+\widetilde{P}) \subset a^{n+1} Q(\widetilde{P}+\widetilde{P}) \subset$ $a^{n}(a \widetilde{P}+a \widetilde{P}) Q \subset a^{n}(P+P) Q \subset P Q \subset Q$. Assume that $a, b \in Q$, say $a \widetilde{P} \subset b \widetilde{P}$, and $b \neq 0$. Then $a+b=\left(a b^{-1}+1\right) b \in(\widetilde{P}+\widetilde{P}) b \subset Q$. So we get $Q+Q \subset Q$.

Corollary 1. Let $K$ be a field with a prime $P$. Then $P_{\circ} \subset P^{\circ}$, and there is no other prime between $P_{\circ}$ and $P^{\circ}$.

Proof. Since $P_{\circ}(P+P) \subset P_{\circ}+P_{\circ}=P_{\circ} \subset P$, we have $P_{\circ} \subset P^{\circ}$. For every prime $Q \subsetneq P^{\circ}$, Proposition 7.4 gives $Q \subset P_{\text {o }}$.

Corollary 2. Let $K$ be a field with a prime $P$. A prime $Q \subset P$ is algebraic if and only if $Q \subset P$.

Proof. Assume that $Q \subset P_{\circ}$. If $Q \neq P_{\circ}$, then $Q \subsetneq P_{\circ} \subset P^{\circ}$, hence $Q$ is algebraic by Proposition 7.4. The converse is trivial.

We call a prime $P$ purely transcendental if $P_{\circ}=0$. If $P \neq 0$, then (P4) implies that $P^{\circ} \neq 0$. By Corollary 1 , this shows that $P^{\circ}$ is minimal if $P \neq 0$ is purely transcendental. Thus Proposition 3.1 gives

Corollary 3. A purely transcendental prime $P \neq 0$ is minimal if and only if $P$ is a Krull prime.

In the paragraph that follows Definition 1.2, we have shown that algebraic primes $P$ of a field $K$ correspond to the valuation rings $\widetilde{P}$ with quotient field $K$. If $P$ is principal, say, $P=p \widetilde{P} \neq 0$, and $\bigcap_{n=0}^{\infty} p^{n} \widetilde{P}=0$, then $\widetilde{P}$ is a discrete valuation domain. Now we turn our attention to compact primes.

Proposition 7.5. A compact Krull prime $P$ of a field $K$ is either algebraic or purely transcendental.

Proof. Assume that $P$ is not purely transcendental. Then $P_{\circ} \neq 0$. Choose $a \in P_{\circ} \backslash 0$. Then $a \widetilde{P_{\circ}} \subset P_{\circ}$. By Proposition 4.1, $P_{\circ}$ is compact. So there are $a_{1}, \ldots, a_{n} \in K$ with $P_{\circ} \subset \bigcup_{i=1}^{n}\left(a_{i}+a P_{\circ}\right)$, hence $\widetilde{P_{\circ}} \subset a^{-1} P_{\circ} \subset \bigcup_{i=1}^{n}\left(a^{-1} a_{i}+P_{\circ}\right)$. Thus $\left|\widetilde{P_{\circ}} / P_{\circ}\right|<\infty$. By $(60)$, this gives $\widetilde{P_{\circ}} \subset \widetilde{P}+\cdots+\widetilde{P}$ 
with finitely many summands. Suppose that $P \neq P_{\circ}$. Then there exist $b \in P \backslash P_{\circ}$ and $n \in \mathbb{N}$ with $b^{n}(P+P) \subset P$. This gives $b^{n+1}(\widetilde{P}+\widetilde{P}) \subset b^{n}(b \widetilde{P}+b \widetilde{P}) \subset P$. Hence, for a suitably large $m \in \mathbb{N}$, we get $b^{m} \widetilde{P_{\circ}} \subset b^{m}(\widetilde{P}+\cdots+\widetilde{P}) \subset P$. If $b^{-m} \in \widetilde{P_{\circ}}$, then $1=b^{m} \cdot b^{-m} \in b^{m} \widetilde{P_{\circ}} \subset P$, a contradiction. Hence $b^{-m} \notin \widetilde{P_{\mathrm{o}}}$, and thus $b^{m} \in P_{\mathrm{o}}$. So we obtain $b \in P_{\mathrm{o}}$, contrary to our assumption. Thus $P=P_{\mathrm{o}}$.

Proposition 7.6. For an algebraic prime $P \neq 0$ of a field $K$, the following are equivalent.

(a) $P$ is compact and minimal.

(b) $P$ is a compact Krull prime.

(c) $\widetilde{P}$ is a discrete valuation domain with finite resudue field.

Proof. The implication (a) $\Rightarrow$ (b) follows by Proposition 3.1 .

(b) $\Rightarrow$ (c): Assume that $a \in P \backslash 0$. Then there exist $a_{1}, \ldots, a_{n} \in K$ with $P \subset \bigcup_{i=1}^{n}\left(a_{i}+\right.$ $\left.a^{2} P\right)$, hence $\widetilde{P} \subset a^{-1} P \subset \bigcup_{i=1}^{n}\left(a^{-1} a_{i}+a P\right)$. Thus $|\widetilde{P} / a P|<\infty$. So we get $|\widetilde{P} / P|<\infty$, and by Proposition 1.1, $P$ is a principal ideal, say $P=p \widetilde{P}$. Since $|\widetilde{P} / a P|<\infty$ holds for all $a \in P \backslash 0$, it follows that $\bigcap_{n=0}^{\infty} P^{n}=0$.

(c) $\Rightarrow\left(\right.$ a): Assume that $a, b \in P \cap K^{\times}$. Then there is some $n \in \mathbb{N}$ with $b \notin P^{n}$. Since $a^{n} \in P^{n}$, we have $b \widetilde{P} \not \subset a^{n} \widetilde{P}$, and thus $a^{n} \widetilde{P} \subset b \widetilde{P}$ by Proposition 1.1. Hence $a^{n+1} \in b P$. Thus, by Proposition 2.2, $P$ is minimal. Assume that $P=p \widetilde{P}$. Then $\left|\widetilde{P} / P^{n}\right|=|\widetilde{P} / P|^{n}$, which implies that $P$ is compact.

Corollary. A compact prime $P \neq 0$ of a field $K$ is a Krull prime if and only if $P$ is minimal.

Proof. Assume that $P$ is a compact Krull prime. If $P$ is purely transcendental, Corollary 1 of Proposition 7.4 implies that $P$ is minimal. Otherwise, $P$ is algebraic by Proposition 7.5, hence minimal by Proposition 7.6. The converse follows by Proposition 3.1.

As already shown in section 6, Proposition 4.1 implies that every non-trivial compact prime $P$ contains a minimal prime.

\section{The product formula}

In this section, we focus our attention upon the minimal compact primes $P$ of a field $K$. If $P$ is algebraic, then Proposition 7.6 implies that $\widetilde{P}$ is a discrete valuation domain with finite residue field. So we have a chain

$$
\cdots \subsetneq P^{2} \subsetneq P \subsetneq P^{0} \subsetneq P^{-1} \subsetneq P^{-2} \cdots
$$

with $P^{0}=\widetilde{P}$ and $P^{n}:=p^{n} \widetilde{P}$. For $a \in K^{\times}$, let $e(a)$ denote the greatest integer $n$ with $a \in P^{n}$. Thus

$$
a \widetilde{P}=P^{e(a)}
$$

holds for any $a \in K^{\times}$. To get the normalized $P$-valuation, we define a function $\chi_{n}: K \rightarrow[0, \infty)$ for any $n \in \mathbb{Z}$ by

$$
\chi_{n}(a):= \begin{cases}1 & \text { for } a \in P^{n} \\ 0 & \text { for } a \notin P^{n}\end{cases}
$$


For $a, b \in K$ with $a-b \in P^{n}$, we have $\left|\chi_{n}(a)-\chi_{n}(b)\right|=0$, which yields $\chi_{n} \in \mathcal{C}_{P}^{+}(K)$.

We set $r_{P}:=|\widetilde{P} / P|$. Then $P^{n} / P^{n+1}=p^{n} \widetilde{P} / p^{n+1} \widetilde{P} \cong \widetilde{P} / p \widetilde{P}$, hence $\left|P^{n} / P^{n+1}\right|=r_{P}$, and thus $\left|P^{n} / P^{n+m}\right|=r_{P}^{m}$ for $m \in \mathbb{N}$. This gives $\chi_{n}: \chi_{n+m}=r_{P}^{m}$, hence

$$
\frac{\chi_{n}: \chi_{n+m}}{\chi_{0}: \chi_{n+m}}=\frac{r_{P}^{m}}{r_{P}^{n+m}}=r_{P}^{-n} .
$$

Therefore, by (48), there is a Haar integral $I$ with $I\left(\chi_{0}\right)=1$ and $I\left(\chi_{n}\right)=r_{P}^{-n}$. For $b \in K^{\times}$, Eq. (62) shows that $\chi_{e(b)}$ is the smallest function in $\mathcal{C}_{P}^{+}(K)$, which takes the value 1 on $b \widetilde{P}$. Now (55) gives $v_{I}(b)=r_{P}^{-e(b)}$, hence $v_{I}(1)=1$. By $(58)$, we get a normalized $P$-valuation

$$
v_{P}(a)=r_{P}^{-e(a)} .
$$

Let us return to the product formula (6) of the introduction. The normalized $P_{p^{-}}$-valuations $v_{p}:=v_{P_{p}}$ for the primes $P_{p}$ of $\mathbb{Q}$ are obtained by (64):

$$
v_{p}(a)=p^{-e(a)}
$$

where $e(a)$ is the exponent of $p$ in a prime factorization of $a$. Thus $v_{p}$ coincides with the $p$-adic absolute value (cf. section 3, Example 1).

The topology of the prime $P_{\infty}=(-1,1) \cap \mathbb{Q}$ of $\mathbb{Q}$ is the usual one. Therefore, a function $f: \mathbb{Q} \rightarrow \mathbb{R}$ belongs to $\mathcal{C}_{\infty}(\mathbb{Q}):=\mathcal{C}_{P_{\infty}}(\mathbb{Q})$ if and only if it is uniformly continuous (in the usual sense) and vanishes outside a bounded interval. The Haar integral with respect to $P_{\infty}$ is just the Riemann-integral. Therefore, the normalized $P_{\infty}$-valuation $v_{\infty}$ can be obtained from (54). In fact, for $f \in \mathcal{C}_{\infty}(\mathbb{Q})$ and $c \in \mathbb{Q}^{\times}$, we get by the substitution $b=c^{-1} a$ :

$$
\int_{\mathbb{Q}} f \rho_{c}(a) d a=\int_{\mathbb{Q}} f\left(c^{-1} a\right) d a=|c| \int_{\mathbb{Q}} f(b) d b .
$$

So the normalized $P_{\infty}$-valuation is the absolute value:

$$
v_{\infty}(a)=|a| .
$$

In the sequel, we denote the set of minimal primes of a field $K$ by $\mathcal{P}(K)$. From (65) and (66) we thus get for all $a \in \mathbb{Q}^{\times}$the product formula:

$$
\prod_{P \in \mathcal{P}(\mathbb{Q})} v_{P}(a)=1
$$

Lemma 8.1. Let $K$ be a field with a minimal prime $P$, and let $a, b \in K^{\times}$be such that $a^{n} \subset b \widetilde{P}$ for all $n \in \mathbb{N}$. Then $a \in \widetilde{P}$.

Proof. Suppose that $a \notin \widetilde{P}$. Then $a^{-1} \in P$. By Proposition 2.2, there is an $n \in \mathbb{N}$ with $a^{-n} \in b^{-1} P$. Hence $1=a^{n} a^{-n} \in b \widetilde{P} b^{-1} P \subset P$, a contradiction!

Proposition 8.1. Every minimal prime of a field $K$ of characteristic $p>0$ is algebraic.

Proof. Let $F$ be the prime field of $K$. Suppose that $a \in F \backslash \widetilde{P}$. Then $a^{-1} \in P$. Thus $\left|F^{\times}\right|=p-1$ implies that $1=\left(a^{-1}\right)^{p-1} \in P$, a contradiction. Therefore, $F \subset \widetilde{P}$. Choose $d \in K^{\times}$with $\widetilde{P}+\widetilde{P} \subset d \widetilde{P}$. 
For $a, b \in \widetilde{P}$ and an arbitrary $n \in \mathbb{N}$ we find some $m \in \mathbb{N}$ with $m n<2^{m}$. Then $(a+b)^{m n}=$ $\sum_{i=0}^{m n}\left(\begin{array}{c}m n \\ i\end{array}\right) a^{i} b^{m n-i} \in \sum_{i=1}^{2^{m}} \widetilde{P} \subset d^{m} \widetilde{P}$. Hence $\left((a+b)^{n} d^{-1}\right)^{m} \in \widetilde{P}$, and thus $(a+b)^{n} d^{-1} \in \widetilde{P}$. By Lemma 3.1, this gives $a+b \in \widetilde{P}$. So $\widetilde{P}+\widetilde{P} \subset \widetilde{P}$. Now Lemma 2.4 implies that $P$ is algebraic.

For a rational prime $p$, let $\mathbb{F}_{p} \cong \mathbb{Z} / p \mathbb{Z}$ denote the field with $p$ elements. Consider the quotient field $\mathbb{F}_{p}(x)$ of $\mathbb{F}_{p}[x]$. By Proposition 8.1, every minimal prime of $\mathbb{F}_{p}(x)$ is algebraic. For any normed irreducible polynomial $q \in \mathbb{F}_{p}[x]$, consider the subring

$$
\mathbb{F}_{p}[x]_{q}:=\left\{\frac{f}{g} \in \mathbb{F}_{p}(x) \mid f, g \in \mathbb{F}_{p}[x], q \nmid g\right\}
$$

of $\mathbb{F}_{p}(x)$. Then $\mathbb{F}_{p}[x]_{q}$ is a discrete valuation ring with residue class field $\mathbb{F}_{p}$. Hence $P_{q}:=q \mathbb{F}_{p}(x)$ is a prime of $\mathbb{F}_{p}(x)$ with $\widetilde{P}_{q}=\mathbb{F}_{p}[x]_{q}$. For all these primes, $\widetilde{P}_{q} \supset \mathbb{F}_{p}[x]$. Furthermore,

$$
P_{\infty}:=x^{-1} \mathbb{F}_{p}\left[x^{-1}\right]_{x^{-1}}
$$

is a prime with $x \notin \widetilde{P_{\infty}}$, hence $\widetilde{P_{\infty}} \not \supset \mathbb{F}_{p}[x]$. In analogy with (15), we write $P_{\infty}$, which does not mean, however, that $P_{\infty}$ is transcendental (which is impossible by Proposition 8.1.

The primes of the form $P_{q}$ with $q$ irreducible, or $q=\infty$, are compact Krull primes by Proposition 7.6. In fact, if $m=\operatorname{deg} q$ is the degree of an irreducible polynomial $q \in \mathbb{F}_{p}[x]$, then $\operatorname{dim}_{\mathbb{F}_{p}}\left(\widetilde{P}_{q} / P_{q}\right)=$ $\operatorname{dim}_{\mathbb{F}_{p}}\left(\mathbb{F}_{p}[x] / q \mathbb{F}_{p}[x]\right)=m$, hence $\left|\widetilde{P_{q}} / P_{q}\right|=p^{m}$. For $P_{\infty}$, we have $\left|\widetilde{P_{\infty}} / P_{\infty}\right|=p$. The normalized $P_{q^{-}}$ valuation $v_{q}:=v_{P_{q}}$ results from (64):

$$
v_{q}(f)=p^{-e_{q}(f) \operatorname{deg} q},
$$

where $e_{q}(f)$ denotes the multiplicity of $q$ in $f$. For example, $e_{x-1}\left(x^{3}-2 x^{2}+x\right)=2$. Moreover, we get

$$
v_{\infty}(f)=p^{\operatorname{deg} f} .
$$

Proposition 8.2. Let $p$ be a rational prime. Every minimal compact prime of $\mathbb{F}_{p}(x)$ is of the form $P_{q}$ with $q$ irreducible, or $q=\infty$.

Proof. Let $P$ be a minimal compact prime of $\mathbb{F}_{p}(x)$. As in the proof of Proposition 8.1, we have $\mathbb{F}_{p} \subset \widetilde{P}$. Assume first that $x \in \widetilde{P}$. Then $\mathbb{F}_{p}[x] \subset \widetilde{P}$. Since $P$ is a prime ideal of $\widetilde{P}$, it follows that $P \cap \mathbb{F}_{p}[x]$ is a prime ideal of $\mathbb{F}_{p}[x]$. If $P \cap \mathbb{F}_{p}[x]=0$, then $a \notin P$ for all $a \in \mathbb{F}_{p}[x] \backslash 0$. Hence $a^{-1} \in \widetilde{P}$, and thus $\mathbb{F}_{p}(x)=\widetilde{P}$, i. e. $P=0$. Consequently, $P \cap \mathbb{F}_{p}[x]$ is a maximal ideal of $\mathbb{F}_{p}[x]$. As $\mathbb{F}_{p}[x]$ is a principal ideal domain, there is an irreducible polynomial $q \in \mathbb{F}_{p}[x]$ with $P \cap \mathbb{F}_{p}[x]=q \mathbb{F}_{p}[x]$. For every $f \in \mathbb{F}_{p}[x]$ with $q \nmid f$, we have $f \notin P$, hence $f^{-1} \in \widetilde{P}$. Therefore, $P_{q}=q \mathbb{F}_{p}[x]_{q} \subset P$. As $P$ is minimal, we obtain $P=P_{q}$. Finally, assume that $x \notin \widetilde{P}$. Then $x^{-1} \in P$. Applying the preceding argument to $\mathbb{F}_{p}\left[x^{-1}\right]$ instead of $\mathbb{F}_{p}[x]$, we get $P_{\infty}=x^{-1} \mathbb{F}_{p}\left[x^{-1}\right]_{x^{-1}} \subset P$. Thus $P_{\infty}=P$.

From (70) and (71) we again obtain the product formula by Proposition 8.2. Note that in both cases, the product formla arises in a natural way: The minimal primes $P$ are all compact, and their normalized $P$-valuations are unique. So the formula describes an intrinsic relation among the minimal primes.

Received: November 2008. Revised: March 2009. 


\section{References}

[1] E. Artin and G. Whaples, Axiomatic characterization of fields by the product formula for valuations, Bull. Amer. Math. Soc. 51 (1945), 469-492

[2] E. Artin and G. Whaples, A note on axiomatic characterization of fields, Bull. Amer. Math. Soc. 52 (1946), 245-247

[3] N. Bourbaki, General topology, Chapters 1-4; Elements of Mathematics, Springer-Verlag, Berlin, 1998

[4] N. Bourbaki, Commutative algebra, Hermann, Paris 1972

[5] D. K. Harrison, Finite and infinite primes for rings and fields, Mem. Amer. Math. Soc. 68 (1966)

[6] W. Krull, Über geschlossene Bewertungssysteme, J. Reine Angew. Math. 190 (1952), 75-92

[7] W. KRulL, Ordnungsfunktionen und Bewertungen von Körpern, Math. Z. 77 (1961) 135-148

[8] J. Neukirch, Algebraic number theory, Grundlehren der Mathematischen Wissenschaften, 322. Springer-Verlag, Berlin, 1999

[9] A. Ostrowski, Über einige Lösungen der Funktionalgleichung $\psi(x) \cdot \psi(x)=\psi(x y)$, Acta Math. 41 (1916), 271-284

[10] I. Reiner, Maximal orders, London Mathematical Society Monographs, No. 5, Academic Press, London-New York, 1975

[11] P. Roquette, Class field theory in characteristic $p$, its origin and development; Class field theory-its centenary and prospect (Tokyo, 1998), 549-631, Adv. Stud. Pure Math. 30, Math. Soc. Japan, Tokyo, 2001

[12] H. WeBer, Ringtopologien auf $Z$ und $Q$, Math. Z. 155 (1977), 287-298

[13] H. WeBer, Charakterisierung der lokalbeschränkten Ringtopologien auf globalen Körpern, Math. Ann, 239 (1979), 193-205

[14] A. WeIL, L'Intégration dans les groupes topologiques et ses applications, Publ. Inst. Math. Univ. Strasbourg, Hermann, Paris, 1965 